\title{
The chemical connection between damped Lyman- $\alpha$ systems and Local Group dwarf galaxies
}

\author{
Á. Skúladóttir ${ }^{1}$, S. Salvadori ${ }^{2,3,4}$, M. Pettini ${ }^{5}$, E. Tolstoy ${ }^{6}$, and V. Hill ${ }^{7}$ \\ ${ }^{1}$ Max-Planck-Institut für Astronomie, Königstuhl 17, 69117 Heidelberg, Germany \\ e-mail: skuladottir@mpia-hd.mpg.de \\ ${ }^{2}$ Dipartimento di Fisica e Astronomia, Università di Firenze, Via G. Sansone 1, Sesto Fiorentino, Italy \\ ${ }_{3}^{3}$ INAF/Osservatorio Astrofisico di Arcetri, Largo E. Fermi 5, Firenze, Italy \\ ${ }^{4}$ GEPI, Observatoire de Paris, PSL Research University, CNRS, Place Jules Janssen, 92190 Meudon, France \\ ${ }^{5}$ Institute of Astronomy, Madingley Road, Cambridge CB3 OHA, England \\ ${ }^{6}$ Kapteyn Astronomical Institute, University of Groningen, PO Box 800, 9700AV Groningen, The Netherlands \\ ${ }^{7}$ Laboratoire Lagrange, Université de Nice Sophia Antipolis, CNRS, Observatoire de la Côte d'Azur, CS34229, 06304 Nice Cedex \\ 4, France
}

Received 24 November 2017 / Accepted 19 February 2018

\begin{abstract}
Abundances of the volatile elements $\mathrm{S}$ and $\mathrm{Zn}$ have now been measured in around 80 individual stars in the Sculptor dwarf spheroidal galaxy, covering the metallicity range $-2.4 \leq[\mathrm{Fe} / \mathrm{H}] \leq-0.9$. These two elements are of particular interest as they are not depleted onto dust in gas, and their ratio, [S/Zn], has thus commonly been used as a proxy for $[\alpha / \mathrm{Fe}]$ in Damped Lyman- $\alpha$ systems (DLAs). The $\mathrm{S}$ abundances in Sculptor are similar to other $\alpha$-elements in this galaxy, consistent with $\mathrm{S}$ being mainly created in core-collapse supernovae, but also having some contribution from type Ia supernovae. However, our results show that $\mathrm{Zn}$ and Fe do not trace all the same nucleosynthetic production channels. In particular, (contrary to $\mathrm{Fe}$ ) $\mathrm{Zn}$ is not significantly produced by type Ia supernovae. Thus, $[\mathrm{S} / \mathrm{Zn}]$ cannot be reliably used as a proxy for $[\alpha / \mathrm{Fe}]$. We propose $[\mathrm{O} / \mathrm{S}]$ as a function of $[\mathrm{S} / \mathrm{H}]$ as a possible alternative. At higher metallicities, the values of [S/Zn] measured in DLAs are inconsistent with those in local dwarf galaxies, and are more compatible with the Milky Way disk. Low-metallicity DLAs are, however, consistent with the most metal-poor stars in Local Group dwarf spheroidal galaxies. Assuming that the dust depletions of $\mathrm{S}$ and $\mathrm{Zn}$ are negligible, our comparison indicates that the star formation histories of DLAs are on average different from both the Milky Way and the Sculptor dwarf spheroidal galaxy.
\end{abstract}

Key words. stars: abundances - ISM: abundances - Local Group - galaxies: ISM - galaxies: abundances - galaxies: dwarf

\section{Introduction}

Connecting the near and the far has always been one of astronomy's main challenges. When it comes to star formation, in the Local Group we are able to resolve individual stars with spectroscopy and/or photometry and thus study the time-dependent details of stellar populations in precise detail. However, as we go further in distance and back in time, such studies quickly become too time consuming or impossible with current astronomical instrumentation. Instead, we are limited to analyzing the integrated light of a whole galaxy to gather information on the history of its stellar populations. Even then, only the brightest galaxies are visible, while dwarf galaxies are generally expected to become too faint to observe with both current and planned instrumentation (e.g., Pettini \& Cooke 2014; Wise et al. 2014; Boylan-Kolchin et al. 2015). Luckily, intervening absorption systems can be observed in the light of bright background sources (usually quasars - QSO - or gamma-ray bursts - GRB), up to very high redshifts, $z \approx 6$ and beyond (e.g., Rafelski et al. 2014; Becker et al. 2015).

The heavy end of the spectrum of such systems are the damped Lyman- $\alpha$ systems (DLAs), defined to have column densities of neutral hydrogen $N(\mathrm{H} \mathrm{I}) \geq 2 \times 10^{20} \mathrm{~cm}^{-2}$. At these high column densities, the gas is self-shielding from ionizing radiation, and thus the hydrogen is mostly neutral, while in other classes of QSO absorption systems a significant fraction of the gas is ionized (e.g., Bechtold 2003). Furthermore, it has been shown that DLAs contain the majority of neutral hydrogen in the universe at all redshifts (e.g., Wolfe et al. 2005; Neeleman et al. 2016; Sánchez-Ramírez et al. 2016), and since stars form from cold neutral clouds and not warm ionized gas (e.g., Wolfire et al. 2003), DLAs are thought to play an important role in the overall star formation history of the universe (e.g., Wolfe et al. 2005).

Identifying DLA systems is straightforward even in lowresolution spectra (e.g., Mas-Ribas et al. 2017), however, the true nature of the underlying galaxies has proved difficult to constrain. Imaging at low redshift, $z<1$, has revealed both low-luminosity and low-surface-brightness galaxies, as well as brighter spiral galaxies (e.g., Krogager et al. 2017 and references therein). Thus, DLAs seem to arise in a wide range of galaxies, the common requirement being a high surface density of neutral gas (Pontzen et al. 2008; Fumagalli et al. 2015; Bird et al. 2015).

With increasing redshift, there is a moderate effect of decreasing mean metallicity in the observed sample of DLA systems, going from $\langle[\mathrm{M} / \mathrm{H}]\rangle \gtrsim-1$ at $z=1$, to $\langle[\mathrm{M} / \mathrm{H}]\rangle \approx-2$ at $z=5$ (Rafelski et al. 2012, 2014). However, at any given redshift there is also a large scatter, exceeding the measurement errors. In the redshift range where DLA measurements are most abundant, $2 \leq z \leq 4$, this scatter covers up to 2 dex in gas metallicity (Rafelski et al. 2012, 2014; Jorgenson et al. 2013), thus indicating 
that DLAs are not a uniform population of galaxies evolving in a homogeneous manner.

In particular, a link has been proposed between the metalpoor DLA population and Local Group dwarf galaxies (e.g., Salvadori \& Ferrara 2012; Cooke et al. 2015; Berg et al. 2015). For nearby galaxies there is a well known mass-metallicity relation: the more massive a galaxy, the higher its metallicity. This holds true whether the metallicities are measured in gas phase (Skillman et al. 1989; Lee et al. 2006) or stars (Kirby et al. 2013). A comparable relation has been shown to exist in DLAs, where the least massive systems are those with the lowest metallicities (Péroux et al. 2012; Christensen et al. 2014; Krogager et al. 2017).

In principle, abundance measurements in DLAs are relatively simple, as most of the gas is neutral and atoms are in a single dominant ionization stage and the ionization corrections are therefore negligible. However, one of the major challenges comes from the fact that a fraction of the metals in the interstellar medium (ISM) is expected to be depleted onto dust (Savage \& Sembach 1996; Jenkins 2009; Jenkins \& Wallerstein 2017; De Cia et al. 2016). Therefore, the abundance measurements of refractory elements, such as $\mathrm{Fe}$ and $\mathrm{Cr}$, in DLAs underestimate their true values, and uncertain dust corrections need to be applied. However, the more volatile elements, in particular $\mathrm{N}, \mathrm{O}, \mathrm{S}$, and $\mathrm{Zn}$, are normally not significantly depleted onto dust at the metallicities and $\mathrm{H}$ I column densities of most DLAs (e.g., Tchernyshyov et al. 2015; De Cia et al. 2016; Jenkins \& Wallerstein 2017), and are therefore especially important tracers of the chemical properties of DLA systems, and absorption systems in general.

Two main contributors of metals in galaxies are core-collapse supernovae ( $\mathrm{SN}$; such as type II), that explode on short time scales $\left(\sim 10^{6-7} \mathrm{yr}\right)$ and enrich the environment primarily with $\alpha$-elements, such as $\mathrm{O}, \mathrm{Mg}, \mathrm{Si}$, and $\mathrm{S}$ (Nomoto et al. 2013). On the other hand, type Ia SN release mainly Fe-peak elements and typically explode around 1-2 Gyr after the onset of star formation (e.g., Matteucci \& Greggio 1986; Wyse \& Gilmore 1988, de Boer et al. 2012). The abundance ratios of $[\alpha / \mathrm{Fe}]$ thus provide information about the relative fractions of type Ia and II SN in a galaxy, as well as the time scales of star formation (e.g., Wheeler et al. 1989).

Therefore it would be useful to have a way of measuring $[\alpha / \mathrm{Fe}]$ in DLAs, preferably using volatile elements to avoid uncertain corrections to account for dust depletion. Out of these, only $\mathrm{O}$ and $\mathrm{S}$ are $\alpha$-elements. The O I line at $1302 \AA$, which is typically used for $\mathrm{O}$ abundance measurements in DLAs, is very strong, and becomes too saturated for accurate measurements at $[\mathrm{O} / \mathrm{H}] \gtrsim-1.5$. At higher metallicities, $\mathrm{S}$ is often the only tracer of $\alpha$-elements in DLA systems. The volatile element $\mathrm{Zn}$ is the heaviest of the iron-group. In the Milky Way, $[\mathrm{Zn} / \mathrm{Fe}]$ is fairly constant over a large range in metallicities, $-2 \lesssim[\mathrm{Fe} / \mathrm{H}] \lesssim 0$, which has prompted the use of $\mathrm{Zn}$ as a proxy for Fe in DLA systems (e.g., Pettini et al. 1990; De Cia et al. 2016) and thus traditionally [S/Zn] has been used as a tracer for $[\alpha / \mathrm{Fe}]$.

Measuring $\mathrm{S}$ and $\mathrm{Zn}$ in stars in Local Group dwarf galaxies poses a new set of challenges. At the distances of the Milky Way dwarf satellites it is only feasible to take the high-resolution spectra, necessary for accurate abundance determination, of the brightest red giant branch (RGB) stars. The $\mathrm{Zn}$ abundance measurements are usually made from a $\mathrm{Zn}$ I line at $4810 \AA$, where the flux is relatively low in these RGB stars. At low metallicities, typical for stars in dwarf galaxies, $\mathrm{S}$ is usually measured from the S I triplet at 9213,9228 , and $9238 \AA$, a region that is relatively poor in atomic lines and thus seldom included when
Table 1. Stellar and dynamical masses of dwarf galaxies discussed in this paper (McConnachie 2012, more details therein).

\begin{tabular}{lccccc}
\hline \hline Galaxy & $\begin{array}{c}M_{\star} \\
10^{6} M_{\odot}\end{array}$ & $\begin{array}{c}M_{d y n} \\
10^{6} M_{\odot}\end{array}$ & $\begin{array}{c}N_{\star} \\
{[\mathrm{Zn} / \mathrm{H}]}\end{array}$ & $\begin{array}{c}N_{\star} \\
{[\mathrm{S} / \mathrm{Zn}]}\end{array}$ & Ref. \\
\hline Sagittarius & 21 & 190 & 11 & 0 & 1 \\
Fornax & 20 & 56 & 2 & 0 & 2 \\
Sextans & 0.44 & 25 & 5 & 0 & 3,4 \\
Sculptor & 2.3 & 14 & 96 & 78 & 2,5 \\
Leo I & 5.5 & 12 & 2 & 0 & 2 \\
Draco & 0.29 & 11 & 9 & 0 & 3,6 \\
Ursa Minor & 0.29 & 9.5 & 21 & 0 & 3,7 \\
Carina & 0.38 & 6.3 & 10 & 0 & 2,8 \\
Bootes I & 0.03 & 0.8 & 3 & 0 & 9 \\
\hline
\end{tabular}

Notes. Also listed are the number of available measurements of $[\mathrm{Zn} / \mathrm{H}]$ and $[\mathrm{S} / \mathrm{Zn}]$ in the literature. Upper limits and stars with abundance errors $>0.5$ dex are not included. References. [1] Sbordone et al. (2007); [2] Shetrone et al. (2003); [3] Shetrone et al. (2001); [4] Honda et al. (2011); [5] Geisler et al. (2005), Kirby \& Cohen (2012), Simon et al. (2015), Skúladóttir et al. (2015a,b, 2017), and Hill et al. (in prep.); [6] Cohen \& Huang (2009); [7] Sadakane et al. (2004), Cohen \& Huang (2010), and Ural et al. (2015); [8] Venn et al. (2012); and [9] Gilmore et al. (2013).

optical spectra of stars are acquired. In addition, these lines are sensitive to departures from local thermodynamical equilibrium (LTE), so non-LTE effects need to be taken into account in the derivation of the $\mathrm{S}$ abundance (Takeda et al. 2005; Korotin 2008; Skúladóttir et al. 2015a).

The chemical properties of the oldest stellar populations in nearby dwarf galaxies offer a direct insight into the star formation processes at early times, comparable to the redshift where most DLAs are observed. Because of these challenges, however, a moderately large sample of S and Zn measurements in stars of a dwarf galaxy has only recently become available in the Sculptor dwarf spheroidal (dSph) galaxy (Skúladóttir et al. 2015a, 2017). Thus for the first time we are able to make a direct comparison of the $[\mathrm{S} / \mathrm{Zn}]$ ratios between DLA systems and stars in dwarf galaxies, without using any other element as a proxy.

\section{Metallicity comparison}

The available $[\mathrm{Zn} / \mathrm{H}]$ abundance measurements of stars in the Local Group and gas in DLAs are shown in Fig. 1 as a function of age/look-back time and redshift. ${ }^{1}$ The conversion between redshift and look-back time was made using Wright $(2006)^{2}$. The measurement errors on the DLA redshifts are negligible compared to the uncertainty of the individual stellar ages, $\langle\Delta \mathrm{Age}\rangle_{\text {Scl }}=\langle\Delta \mathrm{Age}\rangle_{\mathrm{Car}}=1.8 \mathrm{Gyr}$, but some systematic uncertainty in the conversion between redshift and age may remain.

Measurements of $\mathrm{Zn}$ from four Milky Way dSph satellites are shown in Fig. 1. Out of these, the Carina dSph is the smallest and Sagittarius is the largest and most metal-rich; see Table 1. The individual stars with $[\mathrm{Zn} / \mathrm{H}]$ measurements in Draco and in the main body of Sagittarius do not have age determinations. However, the stellar population in Draco is predominantly old

1 The solar abundances throughout this paper are adopted from Grevesse \& Sauval (1998), with the exception of $\log \epsilon(S)_{\odot}=7.16$ (Caffau et al. 2011).

2 The default values of Wright (2006) are assumed $\left(H_{0}=69.6, \Omega_{M}=\right.$ $\left.0.286, \Omega_{\text {vac }}=0.714\right)$, but adopting those determined by the Planck mission (Planck Collaboration XIII 2016) would make very little difference for our present purposes. 


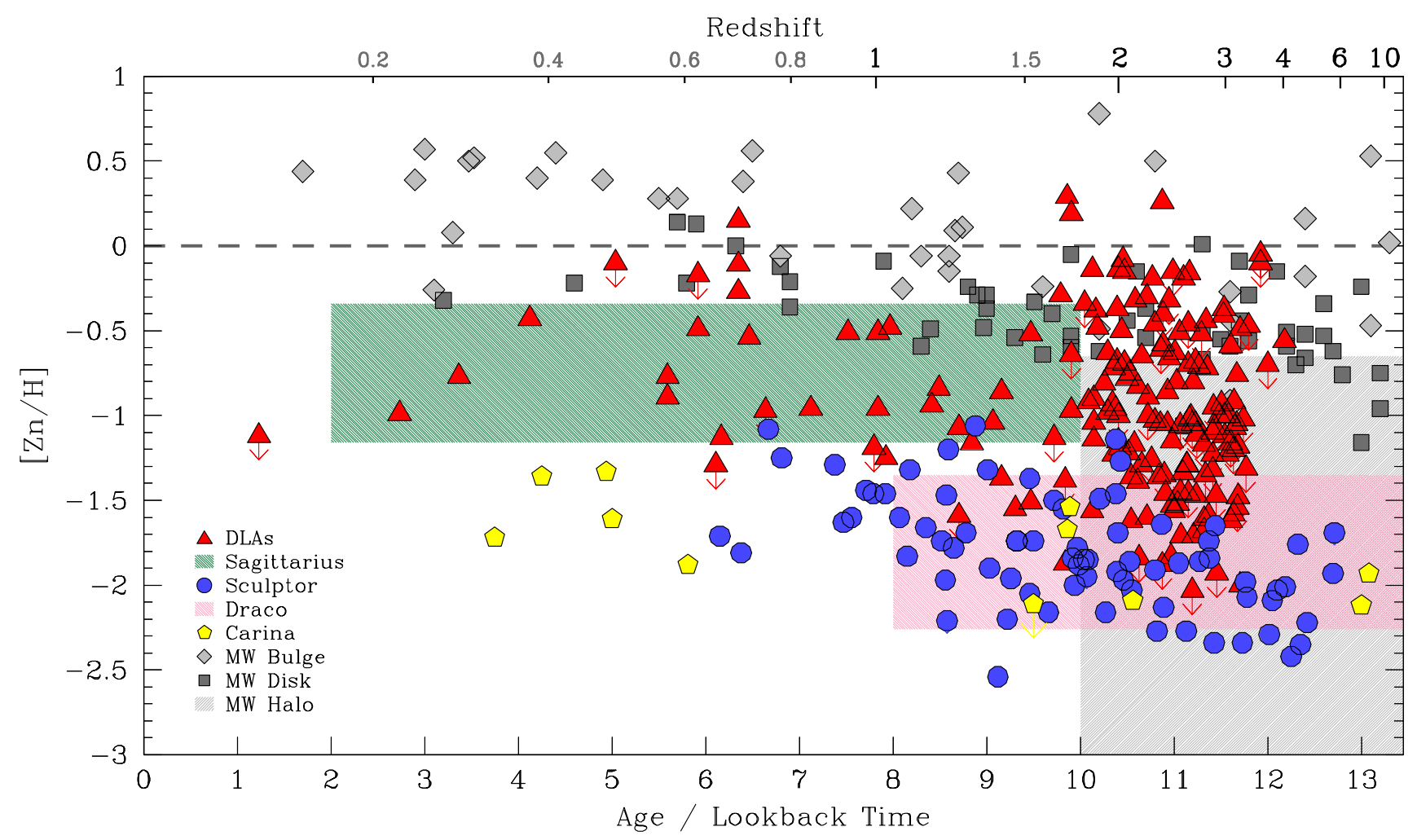

Fig. 1. Zinc abundances as a function of stellar ages/look-back time (lower $x$-axis), and redshift (upper). Red triangles are DLAs (compilation from Quiret et al. 2016), blue circles are Sculptor stars (Skúladóttir et al. 2017; ages from de Boer et al. 2012), and yellow pentagons are Carina stars (Shetrone et al. 2003; Venn et al. 2012; ages from Lemasle et al. 2012). Colored regions show the area occupied by available measurements: Draco in pink (Shetrone et al. 2001; Cohen \& Huang 2009); and Sagittarius in dark green (Sbordone et al. 2007). The Milky Way is shown in gray: diamonds are the bulge (Bensby et al. 2013), squares are the disk (Reddy et al. 2006) and the halo is shown with a shaded area (Cayrel et al. 2004; Reddy et al. 2006; Nissen et al. 2007).

(e.g., Aparicio et al. 2001). The star formation history of Sagittarius is more extended, and the available $\mathrm{Zn}$ measurements are in metal-rich stars, which are expected to be of young and/or intermediate age (Bellazzini et al. 2006; Siegel et al. 2007; de Boer et al. 2015). The range of age and $[\mathrm{Zn} / \mathrm{H}]$ covered by various components of the Milky Way are also shown in gray in Fig. 1. In the case of the bulge and disk, the surveys included age measurements, but for the halo an age range of 10-14 Gyr is assumed (e.g., Salvadori et al. 2010; Carollo et al. 2016).

The information in Fig. 1 is limited to the available data, so it does not necessarily give a complete overview of the star formation histories of the dwarf galaxies in question. In particular, the Sculptor dSph stellar sample is from the center of this galaxy which is younger and more metal-rich compared to other regions. From the star formation history it is estimated that over $80 \%$ of Sculptor's stellar population was formed more than $10 \mathrm{Gyr}$ ago (de Boer et al. 2012), while the data are spread over a larger range of ages, from 6 to 12 Gyr. For Sagittarius, $[\mathrm{Zn} / \mathrm{H}]$ measurements are not available in the older and more metal-poor component.

Most of the DLA data are concentrated in the redshift range $1.5<z<4$; see Fig. 1, which is when star formation in the Universe was at its peak (Madau \& Dickinson 2014). This corresponds to a look-back time of 9.4-12.2 Gyr, when star formation was active in all of the galaxies depicted in Fig. 1. At redshifts of $z \lesssim 1.5$, and $z \gtrsim 4[\mathrm{Zn} / \mathrm{H}]$ becomes very challenging to measure from the ground.

The DLAs span a larger $[\mathrm{Zn} / \mathrm{H}]$ range than any of the individual dwarf galaxies, as they are most probably a mixed bag of different types of systems, at different evolutionary stages (Pettini 2004; Pontzen et al. 2008; Fumagalli et al. 2015,
Krogager et al. 2017). The lower-metallicity DLAs, at $-2 \lesssim$ $[\mathrm{Zn} / \mathrm{H}] \lesssim-1$, are most compatible with the metallicities of dwarf galaxies such as Sculptor, Carina, and Draco, and the more metal-rich part of the Milky Way halo. The more metal-rich DLAs, $[\mathrm{Zn} / \mathrm{H}] \gtrsim-1$, fall in the same range as the Milky Way disk and bulge, and larger dwarf galaxies such as Sagittarius. At the lowest metallicities, $[\mathrm{Zn} / \mathrm{H}] \lesssim-2.5, \mathrm{Zn}$ becomes very challenging to measure, both in stars and DLAs. However, DLAs with undetectable $\mathrm{Zn}$ lines only amount to a small fraction of the population in this redshift range.

\section{Chemical abundance ratios with $\mathrm{Fe}$}

\section{1. $[\mathrm{S} / \mathrm{Fe}]$ and $[\mathrm{Zn} / \mathrm{Fe}]$}

The available abundances of the key elements Fe, S, and Zn, measured in gas in DLAs and in RGB stars in the Sculptor dSph are shown in Fig. 2. The right panel displays the binned data, also including measurements of dwarf stars in the Milky Way. DLA systems are limited to $z>1.5$ because of observational difficulties at lower redshifts, and also because this corresponds to the epoch where the majority of Sculptor stars were formed $(\approx 90 \%$ according to de Boer et al. 2012). When comparing these abundance ratios, it is important to remember that DLA abundances are measurements along one line of sight in the ISM of a (proto)galaxy, while Sculptor and Milky Way abundances come from individual stars. The binned data for DLAs therefore represent averages over many galaxies, while the stellar abundances are averages for stars within the same galaxy. 

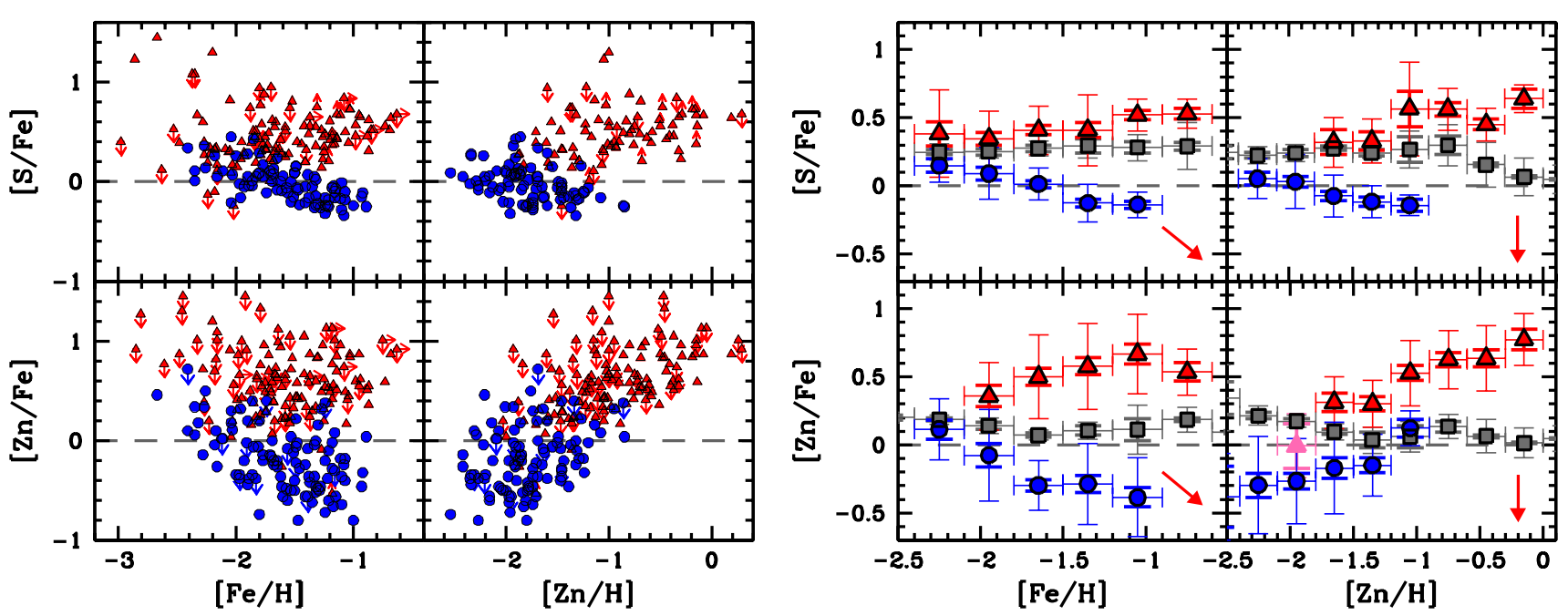

Fig. 2. Left panel: abundance ratios of $[\mathrm{S} / \mathrm{Fe}]$ and $[\mathrm{Zn} / \mathrm{Fe}]$ in DLAs with $z<1.5$ (red triangles) and Sculptor stars (blue circles) as a function of $[\mathrm{Fe} / \mathrm{H}]$ and $[\mathrm{Zn} / \mathrm{H}]$. Right panel: same with binned data, excluding upper/lower limits. Also included are Milky Way dwarf stars as gray squares. The size of each bin is shown with $x$-axis error bars. The thick $y$-axis error bars show the error of the mean in each bin, while thin show $1 \sigma$ of the scatter. The pink triangle only includes three DLAs. Red arrows show the direction (but not necessarily the size) of dust depletion corrections that should be applied to DLA abundances. We note that the range of both the $x$ - and $y$-axis is not the same on the left and right panels. References: DLAs: Compilation by Quiret et al. (2016); Sculptor: Skúladóttir et al. (2017) (Zn), Skúladóttir et al. (2015a) (S), Skúladóttir et al. (2015b) (1 star); Milky Way: Reddy et al. (2003, 2006), Nissen et al. (2007).

From these data it is evident that the Sculptor dSph, the Milky Way, and DLAs all show clear differences in their $[\mathrm{S} / \mathrm{Fe}]$ and $[\mathrm{Zn} / \mathrm{Fe}]$ ratios, both as a function of $[\mathrm{Fe} / \mathrm{H}]$ and $[\mathrm{Zn} / \mathrm{H}]$. In addition, Sculptor stars cover a lower $[\mathrm{Zn} / \mathrm{H}]$ range compared to DLAs. This implies that stars in Sculptor are typically more metal-poor than gas in DLAs, even when observations are limited to the same age/redshift range (see also Fig. 1). On the other hand, when looking at $[\mathrm{Fe} / \mathrm{H}]$ abundances, we might have the misguided impression that DLAs cover the same metallicity range as stars in Sculptor. This is due to the fact that a fraction of Fe in DLAs is depleted onto dust and therefore not detected. Hence, it is extremely important to use volatile elements such as $\mathrm{S}$ and $\mathrm{Zn}$ when comparing metallicities in stars and gas.

The dust depletion of $\mathrm{Fe}$ also affects the $[\mathrm{S} / \mathrm{Fe}]$ and $[\mathrm{Zn} / \mathrm{Fe}]$ abundances, and the arrows in Fig. 2 show the direction of dust depletion corrections that should be applied in each case. However, the size of these corrections is not well constrained but should be smaller at lower metallicities, because of less efficient dust formation (e.g., Vladilo 2002; Akerman et al. 2005; Jenkins \& Wallerstein 2017). Depending on the level of dust depletion, the DLA $[\mathrm{S} / \mathrm{Fe}]$ or $[\mathrm{Zn} / \mathrm{Fe}]$ abundance ratios could therefore resemble those of the Milky Way, or Sculptor, or neither.

\subsection{Can we quantify dust depletion?}

A standard approach to correct for dust depletion in DLAs is to assume that $[\mathrm{Zn} / \mathrm{Fe}]$ ratios in DLAs are intrinsically the same as in the Milky Way stars at any given metallicity. Since S and $\mathrm{Zn}$ are not significantly depleted at the metallicities and $\mathrm{H} \mathrm{I}$ column densities of most DLAs (Jenkins \& Wallerstein 2017), we can quantify the dust depletion correction as $\Delta[\mathrm{Zn} / \mathrm{Fe}]_{\mathrm{MW}}=$ $[\mathrm{Zn} / \mathrm{Fe}]_{\text {DLA }}-[\mathrm{Zn} / \mathrm{Fe}]_{\mathrm{MW}}$ or equivalently by using $[\mathrm{S} / \mathrm{Fe}]$ abundances. On the other hand, if it is assumed that $[\mathrm{Zn} / \mathrm{Fe}]$ or $[\mathrm{S} / \mathrm{Fe}]$ should behave the same in DLAs and the Sculptor dSph, then the dust depletion can be determined using $\Delta[\mathrm{X} / \mathrm{Fe}]_{\mathrm{Scl}}$.

The values of $\Delta[\mathrm{X} / \mathrm{Fe}]_{\mathrm{MW} / \mathrm{Scl}}$ are shown in Fig. 3. It is clear that neither $[\mathrm{Zn} / \mathrm{Fe}]$ nor $[\mathrm{S} / \mathrm{Fe}]$ behave the same way in Milky Way and in Sculptor (see Figs. 2 and 3), showing that these abundance ratios are not a constant throughout all environments, and casting doubt on whether this is a robust method for measuring dust depletion.

Only DLAs and stars that have measurements of all three elements, $\mathrm{Fe}, \mathrm{Zn}$, and $\mathrm{S}$, are shown in Fig. 3 as a function of $[\mathrm{Fe} / \mathrm{H}],[\mathrm{Zn} / \mathrm{H}]$, and $[\mathrm{S} / \mathrm{H}]$. Out of these, the top panel is the most challenging to interpret, since we are not necessarily comparing the same metallicities in stars and DLAs, due to dust depletion of $[\mathrm{Fe} / \mathrm{H}]$ in DLAs, so here we focus on the other two panels. In any given metallicity bin the average differences $\Delta[\mathrm{Zn} / \mathrm{Fe}]$ and $\Delta[\mathrm{S} / \mathrm{Fe}]$ refer to exactly the same set of DLA and stellar measurements. This means that even though different DLAs might suffer different levels of dust depletion, if $[\mathrm{S} / \mathrm{Fe}]$ and $[\mathrm{Zn} / \mathrm{Fe}]$ are both the same as in the Milky Way, the average dust depletion of the bin should be the same whether measured from $\Delta[\mathrm{S} / \mathrm{Fe}]$ or $\Delta[\mathrm{Zn} / \mathrm{Fe}]$, and as a function of either $[\mathrm{S} / \mathrm{H}]$ or $[\mathrm{Zn} / \mathrm{H}]$.

In the lowest panel of Fig. 3, when $[\mathrm{S} / \mathrm{H}]$ is used as a reference element, the values of dust depletion as measured from $\Delta[\mathrm{S} / \mathrm{Fe}]_{\mathrm{MW}}$ and $\Delta[\mathrm{Zn} / \mathrm{Fe}]_{\mathrm{MW}}$ agree within error bars, although the latter is always systematically higher. However, when $[\mathrm{Zn} / \mathrm{H}]$ is used as a reference element (middle panel Fig. 3), this is not the case. This means that there is no dust correction that can be applied to make both the $[\mathrm{S} / \mathrm{Fe}]$ and $[\mathrm{Zn} / \mathrm{Fe}]$ ratios agree between the Milky Way (or Sculptor) and DLA systems at any given metallicity. This can result from some or all of the following effects: i) The chemical enrichment history of the Milky Way (or Sculptor) and the average of DLAs in each metallicity bin is not the same; ii) there is some dust depletion present of $\mathrm{S}$ and/or $\mathrm{Zn}$ in DLAs; iii) the stellar abundances are inaccurate (e.g., due to incomplete stellar physics applied); iv) the dust correction of $\mathrm{Fe}$ is inhomogeneous in each DLA, and correlated with S and/or $\mathrm{Zn}$ abundance in each region along the line of sight, creating this effect.

\subsection{The complications with $\mathrm{Zn}$}

It is clear from both Figs. 2 and 3 that $[\mathrm{Zn} / \mathrm{Fe}]$ is not the same in the Sculptor dSph galaxy and the Milky Way at all 

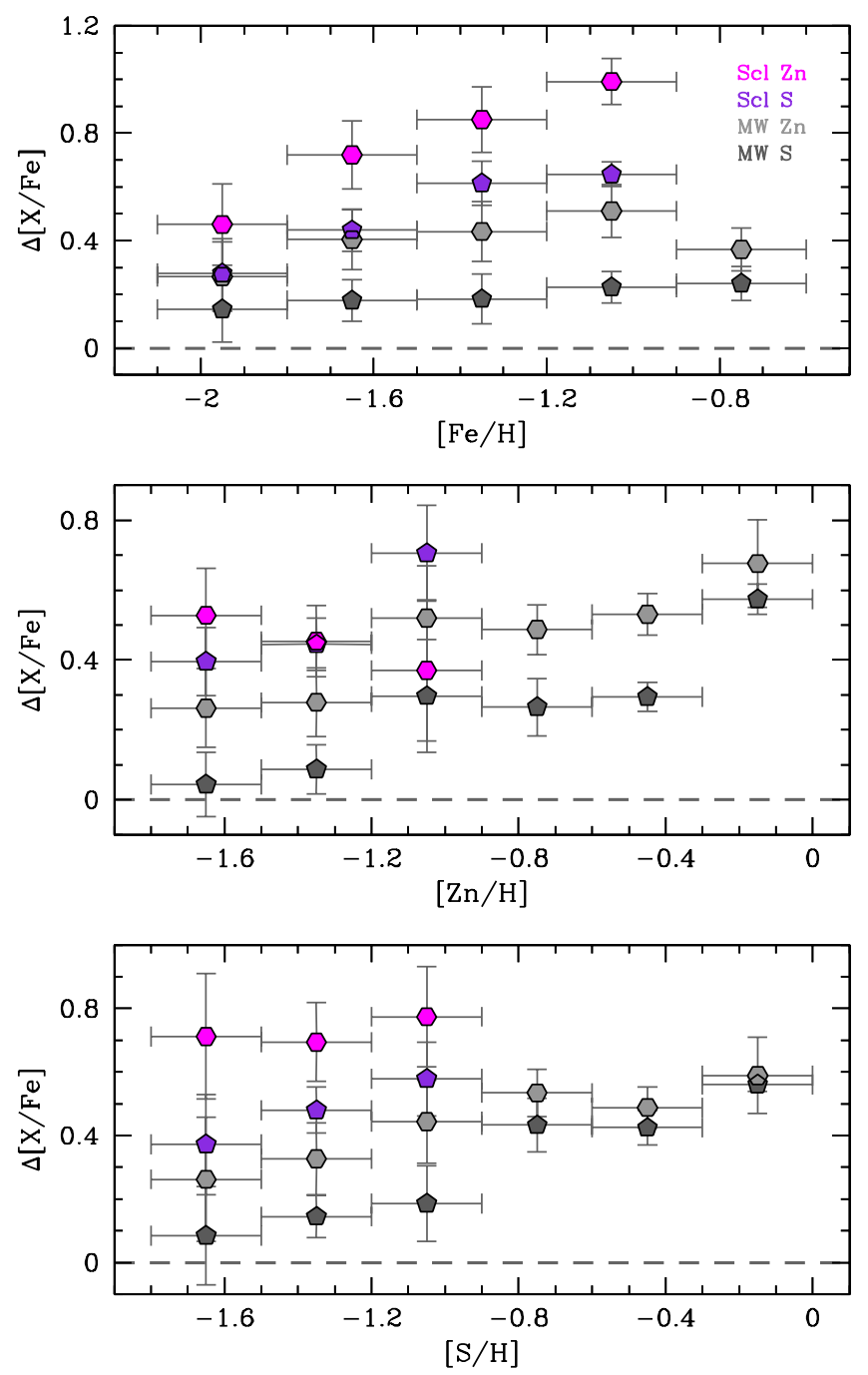

Fig. 3. Differences between measured abundances in DLAs and stars as a function of $[\mathrm{Fe} / \mathrm{H}]$ (top panel), $[\mathrm{Zn} / \mathrm{H}]$ (middle panel) and $[\mathrm{S} / \mathrm{H}]$ (bottom panel). Comparisons with Sculptor $(\mathrm{Scl})$ stars are shown in pink (Zn) and purple (S), and with the Milky Way (MW) stars in light gray ( $\mathrm{Zn})$ and dark gray (S). Hexagons are $\Delta[\mathrm{Zn} / \mathrm{Fe}]$ and pentagons are $\Delta[\mathrm{S} / \mathrm{Fe}]$. Only DLAs and stars that have published measurements of all three elements, $\mathrm{Fe}, \mathrm{S}$, and $\mathrm{Zn}$, are included. The $y$-axis error bars show the quadratic sum of the errors of the means for DLAs and $\mathrm{Scl}$ or MW, while $x$-axis error bars show the size of each bin. Reference: see Fig. 2.

metallicites. The question thus becomes one regarding whether or not $\mathrm{Zn}$ abundances in Sculptor are particularly unusual. A schematic view of the available measurements of stars in Local Group dwarf spheroidal galaxies is shown in Fig. 4, along with the different Milky Way components. It is immediately evident that $[\mathrm{Zn} / \mathrm{Fe}]$ is not constant at all metallicities in any of these galaxies, including the Milky Way. In addition, dwarf galaxies typically exhibit different values of $[\mathrm{Zn} / \mathrm{Fe}]$ at a given $[\mathrm{Fe} / \mathrm{H}]$ compared to the Milky Way. All galaxies (with the possible exception of Carina) seem to show a trend of declining $[\mathrm{Zn} / \mathrm{Fe}]$ with increasing $[\mathrm{Fe} / \mathrm{H}]$. For Sculptor, Draco, Sextans, and Ursa Minor this happens at a similar metallicity, while it is not completely clear where this trend lies in Sagittarus, due to limited data. With the exception of Sculptor, the mean trend in these dwarf galaxies is only determined by 5-21 stars (see Table 1), and should thus only be interpreted as an indication. In particular, the $\mathrm{Zn}$ abundances cannot always be measured at
$[\mathrm{Fe} / \mathrm{H}]<-2.5$. Thus, the rise in $[\mathrm{Zn} / \mathrm{Fe}]$ at the lowest values of $[\mathrm{Fe} / \mathrm{H}]$ in dwarf galaxies is highly uncertain, and may well be less pronounced than it appears in Fig. 4.

To complicate matters even further, there is significant scatter in $[\mathrm{Zn} / \mathrm{Fe}]$ at a given $[\mathrm{Fe} / \mathrm{H}]$ in the Local Group dwarf spheroidal galaxies, which is not well understood; see Fig. 4. The measurement of $\mathrm{Zn}$ in dwarf galaxies is challenging, and can only been done in giant stars, and usually only one line is used. Therefore, some of the scatter in the Sculptor dSph probably reflects measurement uncertainties; however, not all stars at a given $[\mathrm{Fe} / \mathrm{H}]$ are consistent with having the same $[\mathrm{Zn} / \mathrm{Fe}]$ while that is true for most $[\alpha / \mathrm{Fe}]$ measurements in this galaxy (e.g., Skúladóttir et al. 2015a, 2017, Hill et al. in prep). Outliers in $[\mathrm{Zn} / \mathrm{Fe}]$ can also be found in other dwarf galaxies (see Fig. 4), although data is limited.

At the lowest metallicities in the Milky Way, there is an increasing trend of $[\mathrm{Zn} / \mathrm{Fe}]$ with decreasing $[\mathrm{Fe} / \mathrm{H}]$. At the highest metallicities, $[\mathrm{Fe} / \mathrm{H}] \geq-0.3$, there is a discrepancy between $[\mathrm{Zn} / \mathrm{Fe}]$ as measured in dwarf and giant stars in the Milky Way, where the latter show lower values (Bensby et al. 2014; Barbuy et al. 2015; Duffau et al. 2017). There is currently no consensus as to the reason for this discrepancy. One possibility is that it may be due to inadequacies in synthesizing line formation in stellar atmospheres ${ }^{3}$. Perhaps most likely is that dwarf and giant stars at high metallicities probe different populations, as the giant stars are brighter and thus can be observed over greater distances (for a more detailed discussion, see Duffau et al. 2017). In the metallicity range $-2.5 \lesssim[\mathrm{Fe} / \mathrm{H}] \lesssim-0.5$, measurements of $[\mathrm{Zn} / \mathrm{Fe}]$ in dwarf and giant stars in the Milky Way are in very good agreement, meaning that if the discrepancy arises from differences in stellar physics between dwarf and giant stars, these effects are only important at higher metallicities. At the lowest $[\mathrm{Fe} / \mathrm{H}]<-2.5, \mathrm{Zn}$ abundances have currently only been measured in giant stars.

Turning to theory, $\mathrm{Zn}$ is believed to be mainly produced in type II SN and high-energy SN, referred to as hypernovae (Heger \& Woosley 2002; Kobayashi et al. 2006). Other production sites have also been proposed, such as the $s$-process (Travaglio et al. 2004) and neutrino-rich wind of a newly formed neutron star (Pruet et al. 2005), or electron-capture SN (Wanajo et al. 2018; Hirai et al. 2018). The ejecta of type Ia SN, on the other hand, are thought to contain relatively small amounts of $\mathrm{Zn}$, with abundance ratios as low as $[\mathrm{Zn} / \mathrm{Fe}] \lesssim-1.2$, accompanied also by subsolar ratios of $\alpha$-elements, $[\alpha / \mathrm{Fe}]<0$ (Iwamoto et al. 1999; Kobayashi et al. 2006). These type Ia SN start to effect the chemical composition of their environment 1-2 Gyr after onset of star formation and thus lower $[\alpha / \mathrm{Fe}]$ and $[\mathrm{Zn} / \mathrm{Fe}]$ in their surroundings. Since star formation in dwarf galaxies is less efficient than in the Milky Way, this happens at lower $[\mathrm{Fe} / \mathrm{H}]$. The decrease of $[\mathrm{Zn} / \mathrm{Fe}]$, shown in Fig. 4, corresponds to the same trends in $[\alpha / \mathrm{Fe}]$ in each galaxy (Tolstoy et al. 2009 and references therein). Calculations using theoretical yields have also shown that the measured decrease of $[\mathrm{Zn} / \mathrm{Fe}]$ and $[\alpha / \mathrm{Fe}]$ with $[\mathrm{Fe} / \mathrm{H}]$ in the Sculptor dSph galaxy are consistent with being caused by a type Ia SN contribution to the abundance of Fe (Skúladóttir et al. 2017).

In the Milky Way, where stars span a larger range of $[\mathrm{Fe} / \mathrm{H}]$, the evolution of $[\mathrm{Zn} / \mathrm{Fe}]$ is complicated by the fact that the yields of $\mathrm{Zn}$ in type II SN and hypernovae are very metallicity dependent, with a sharp increase in $[\mathrm{Zn} / \mathrm{Fe}]$ at $[\mathrm{Fe} / \mathrm{H}] \gtrsim-1$ (Kobayashi et al. 2006), which counteracts the influence of type Ia SN at a

3 All available calculations for the effects of non-LTE, 3D and granulations are unable to explain this difference, see Duffau et al. (2017). 


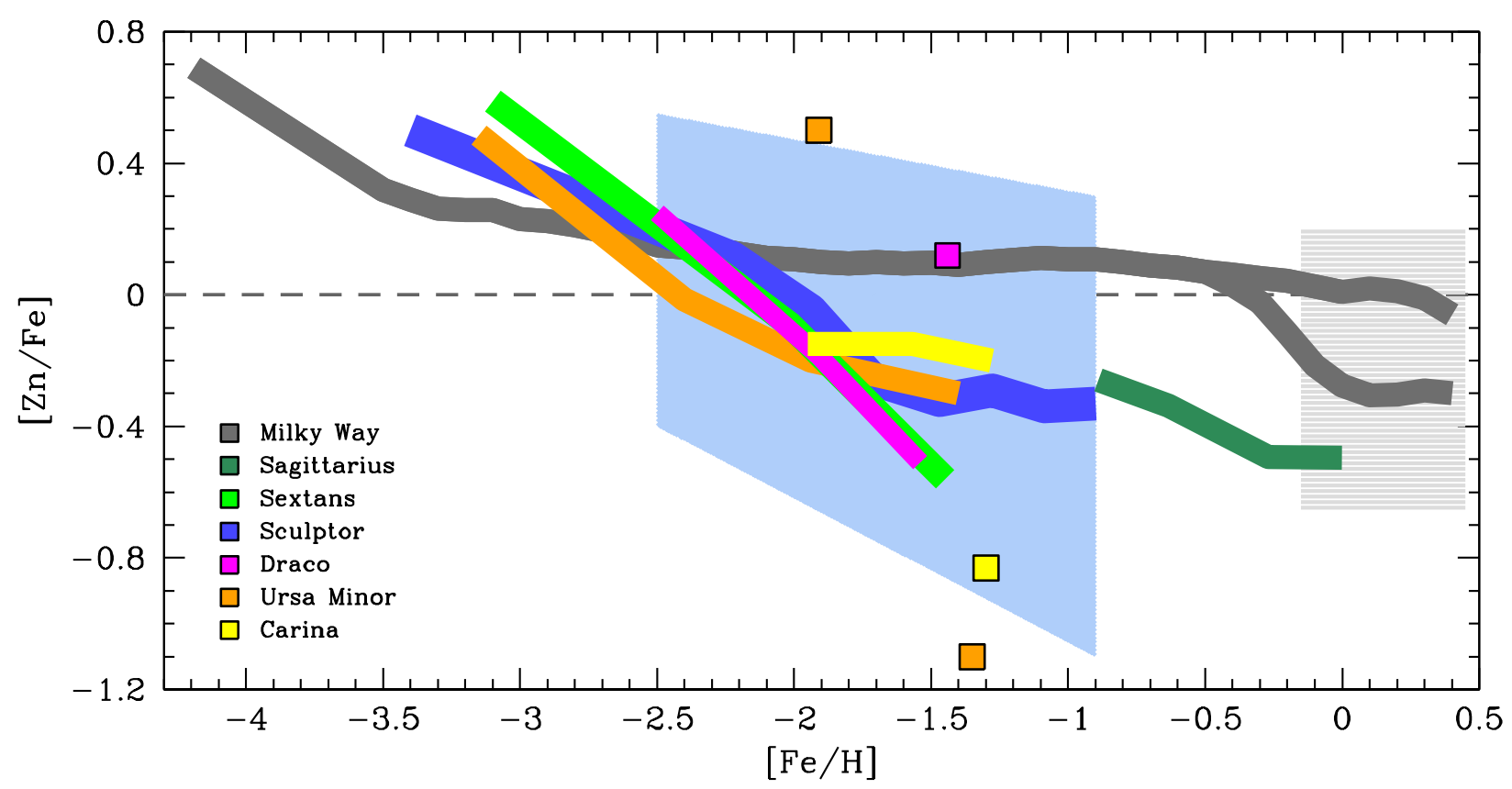

Fig. 4. Schematic figure of $[\mathrm{Zn} / \mathrm{Fe}]$ as a function of $[\mathrm{Fe} / \mathrm{H}]$ in the Milky Way (gray) and its dwarf galaxy satellites: Sagittarius (dark green), Sextans (green), Sculptor (blue), Draco (pink), Ursa Minor (orange) and Carina (yellow). Lines show mean trends, while scatter is indicated by shaded areas, and notable outliers are identified by individual squares. The two branches at $[\mathrm{Fe} / \mathrm{H}] \gtrsim-0.5$ in the Milky Way represent the mean trends as measured by dwarf stars (higher values) and giant stars (lower values). References: Dwarf galaxies: see Table 1. Milky Way: Reddy et al. (2003, 2006), Cayrel et al. (2004), Nissen \& Schuster (2011), Ishigaki et al. (2013), Bensby et al. (2014), Barbuy et al. (2015), and Duffau et al. (2017).

similar metallicity in the Milky Way. This creates the illusion that $\mathrm{Zn}$ and $\mathrm{Fe}$ are co-produced, whereas the fairly constant value of $[\mathrm{Zn} / \mathrm{Fe}]$ at $-2.5<[\mathrm{Fe} / \mathrm{H}]<0$ is the result of the particular star formation history of the Milky Way, and need not apply to other galaxies. The effects of type Ia SN on $[\mathrm{Zn} / \mathrm{Fe}]$ can still be seen in the Milky Way by looking at stars with similar $[\mathrm{Fe} / \mathrm{H}]$ but different $[\alpha / \mathrm{Fe}]$. In these cases $[\mathrm{Zn} / \mathrm{Fe}]$ typically correlates with $[\alpha / \mathrm{Fe}]$ (Nissen \& Schuster 2011; Mikolaitis et al. 2017).

\section{Un-depleted chemical elements}

\section{1. $\mathrm{Zn}$ as a reference element}

The abundance ratios of $[\mathrm{S} / \mathrm{Zn}]$ as a function of $[\mathrm{Zn} / \mathrm{H}]$ are shown for DLAs, the Sculptor dSph, and the Milky Way in Fig. 5. As previously noted, Sculptor stars cover a different $[\mathrm{Zn} / \mathrm{H}]$ range compared to the DLAs. However, where these samples overlap, their $[\mathrm{S} / \mathrm{Zn}]$ abundance ratios are in good agreement, while the Milky Way shows a slightly different trend. A straightforward interpretation could be that the lower metallicity DLAs correspond to systems very similar to the Sculptor dwarf spheroidal galaxy.

However, the complexity of the evolution of $\mathrm{Zn}$ in Local Group galaxies (shown in Fig. 4 and discussed in Sect. 3.3) leads us to question the value of $[\mathrm{Zn} / \mathrm{H}]$ as a reference for metallicity (at least in dwarf galaxies). Ideally, an element used as a proxy for metallicity should be: i) reliably measured; ii) strongly connected to the evolutionary state of the galaxy or its component; iii) of well understood nucleosynthesis. From the point of view of stars in dwarf galaxies, $[\mathrm{Zn} / \mathrm{H}]$ fails on all fronts, as it is difficult to measure, thus having large abundance measurement errors. In addition, there is an intrinsic scatter, which is often difficult to distinguish from uncertainties, and the theoretical understanding of $\mathrm{Zn}$ production in dwarf galaxies is very limited.
Since $\mathrm{Zn}$ does not trace type Ia SN (see Sect. 3.3), and has a large scatter for a given $[\mathrm{Fe} / \mathrm{H}]$ (both intrinsic and due to measurement errors), $[\mathrm{Zn} / \mathrm{H}]$ abundances have a relatively weak correlation to the stellar ages in Sculptor, as shown with color coding in Fig. 4. Thus, any abundance ratios in Sculptor (and other dwarf galaxies) become very difficult to interpret as a function of $[\mathrm{Zn} / \mathrm{H}]$.

\section{2. $S$ as a reference element}

As $\mathrm{Zn}$ is a problematic reference element, we propose the use of $[\mathrm{S} / \mathrm{H}]$ instead. Although $\mathrm{S}$ is an $\alpha$-element, a non-negligible amount is created in type Ia SN. Therefore, S traces both type Ia and type II SN, similar to Fe, but in different proportions. The exact fraction of the contribution from each $\mathrm{SN}$ type depends on the star formation history of the system and the adopted models and yields, but as an example, Tsujimoto et al. (1995) predicted around $25 \%$ of $\mathrm{S}$ in the solar neighborhood to be created by type Ia SN, while the fraction for Fe is around $60 \%$. Therefore, although $\mathrm{S}$ should not be used interchangeably with $\mathrm{Fe}$, these two elements trace the same processes.

Compared to other elements, however, $\mathrm{S}$ is relatively difficult to measure in DLAs, as the available S II lines $(1259,1253$, and $1250 \AA$ ) are weak and in many cases fall within the Lyman- $\alpha$ forest, where blending can be a problem. Other choices, in particular $\mathrm{Si}$, might also be a good reference element for DLAs, but the available Si measurements for stars in the Sculptor dSph are very limited (see Appendix A), and therefore not suitable for our present purpose. In addition, $\mathrm{Si}$ is predicted to suffer more dust depletion compared to S (e.g., De Cia et al. 2016).

The relation between $\mathrm{Zn}$ and $\mathrm{S}$ as a function of $[\mathrm{S} / \mathrm{H}]$ is shown in Fig. 6 for DLAs, the Milky Way, Sculptor and other dwarf galaxies. Since measurements of these elements in other dwarf galaxies are scarce, they are binned together in Fig. 6 using a proxy: $[\mathrm{S} / \mathrm{H}] \approx \frac{1}{2}([\mathrm{Ca} / \mathrm{H}]+[\mathrm{Ti} / \mathrm{H}])$ (see Appendix $\left.\mathrm{A}\right)$, as there 

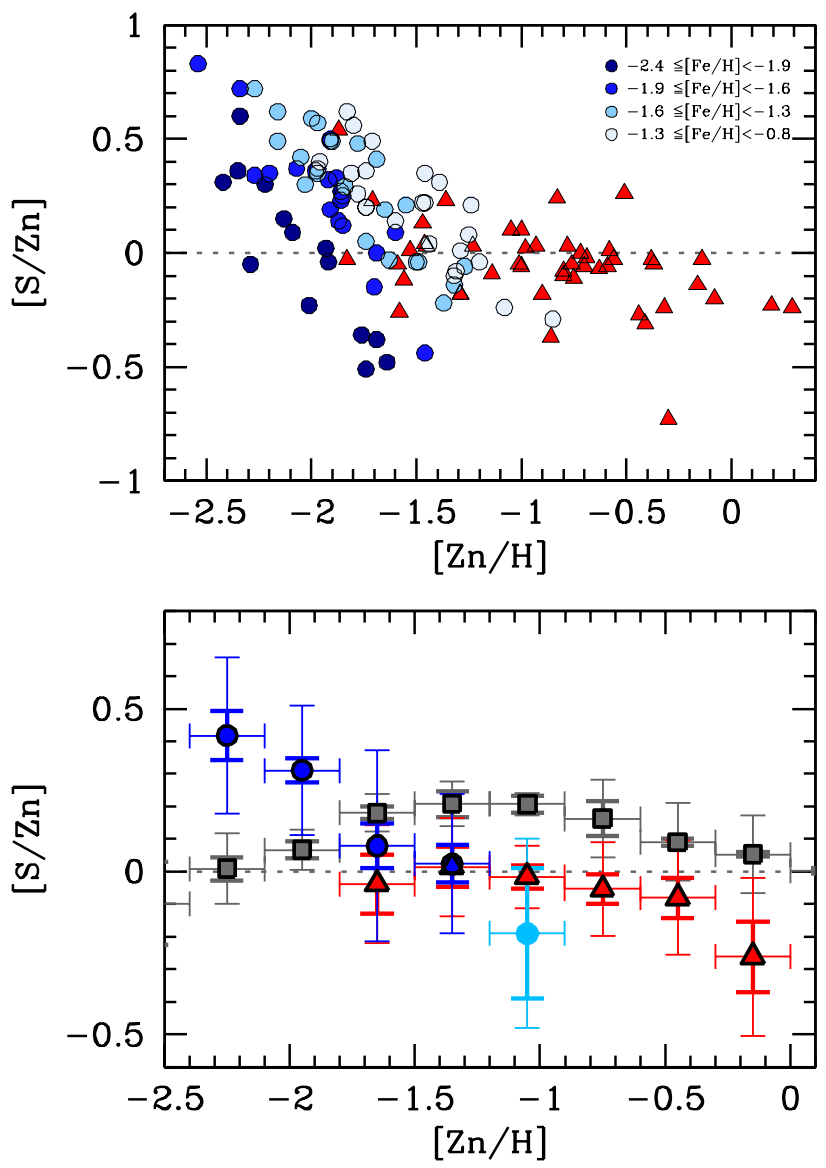

Fig. 5. Abundance ratios of $[\mathrm{S} / \mathrm{Zn}]$ as a function of $[\mathrm{Zn} / \mathrm{H}]$ for DLAs $(z>1.5)$, as well as Sculptor and Milky Way stars. In the top panel, Sculptor stars are circles, colored according to their ages: the oldest stars are dark blue, and the youngest are light blue. Stars without age determination are represented by unfilled circles. The light blue circle in the bottom panel only includes three Sculptor stars. Otherwise, symbols are the same as in Fig. 2, where references are also listed.

are no available $\mathrm{S}$ measurements for these stars. As shown by the average Sculptor stellar ages (de Boer et al. 2012) listed in Fig. 6, $[\mathrm{S} / \mathrm{H}]$ is a reasonable tracer of the evolution of this galaxy. The age- $[\mathrm{S} / \mathrm{H}]$ relation in the other dwarf galaxy sample is less clear, however, as it contains the averages of many different galaxies. In the case of DLAs, there is not a clear age/redshift trend with $[\mathrm{S} / \mathrm{H}]$, as there is a very large metallicity scatter at any redshift (e.g., Rafelski et al. 2012, 2014), but the DLA sample in Fig. 6 is limited to $z>1.5$ (age $>9.4 \mathrm{Gyr}$ ).

The trend of increasing $[\mathrm{S} / \mathrm{Zn}]$ with $[\mathrm{S} / \mathrm{H}]$ in Sculptor stars can be interpreted as resulting from an added contribution to the $\mathrm{S}$ abundance by type Ia SN which produce very small amounts of Zn compared to S (Iwamoto et al. 1999; Kobayashi et al. 2006). For a more detailed discussion and calculations of this, see Skúladóttir et al. (2017). The ratio [S/Zn] appears to "saturate" at $\approx+0.2$ dex at $[\mathrm{S} / \mathrm{H}]>-1.5$, that is, for stars with ages $<10 \mathrm{Gyr}$. The reason for this behavior is not well understood; possibly it may be caused by a metallicity dependence of $\mathrm{Zn}$ yields in type II $\mathrm{SN}$, or other unidentified processes adding to the $\mathrm{Zn}$ production, such as neutrino-driven winds (Hoffman et al. 1996) or the weak and/or main $s$-processes (Mishenina et al. 2002; Travaglio et al. 2004).

The behavior of $[\mathrm{S} / \mathrm{Zn}]$ versus $[\mathrm{S} / \mathrm{H}]$ seen in the other Local Group dwarf galaxies considered here seems on average to be similar to that seen in Sculptor (see Fig. 6). However, we note that this result might be biased, due to lack of data. With the exception of the most $[\mathrm{S} / \mathrm{H}]$-rich bin, the data shown in Fig. 6 are dominated by dwarf galaxies of similar size or smaller than the Sculptor dSph (see Table 1). If $\mathrm{Zn}$ behaves similarly to $\alpha$-elements in the larger dwarf galaxies, Sagittarius and Fornax (as $\mathrm{Zn}$ does in the smaller ones), subsolar [S/Zn] are expected at higher $[\mathrm{S} / \mathrm{H}]$ compared to Sculptor and the sample shown in Fig. 6.

Turning to the Milky Way, we see in Fig. 6. a very complex structure in $[\mathrm{S} / \mathrm{Zn}]$ versus $[\mathrm{S} / \mathrm{H}]$. In halo stars, $[\mathrm{S} / \mathrm{Zn}]$ decreases with decreasing $[\mathrm{S} / \mathrm{H}]$, which has been interpreted as evidence for $\mathrm{Zn}$ production in hypernovae, which can produce large amounts of Zn (Umeda \& Nomoto 2002). Disk stars seem to be disjointed from this behavior in the halo, and more similar to DLAs. The lower values of $[\mathrm{S} / \mathrm{Zn}]$ when $[\mathrm{S} / \mathrm{H}]>-1$ in the Milky Way disk have been explained by more efficient $\mathrm{Zn}$ production in type II SN and hypernovae at higher metallicities (Kobayashi et al. 2006; Nomoto et al. 2013).

Given the complexity of the [S/Zn] ratio in different Local Group stellar populations, it is remarkable to see in Fig. 6 an essentially constant mean value at all the metallicities probed by DLAs. In particular since the DLAs here are expected to cover a wide range in mass. By adopting the mass-metallicity relation of Christensen et al. (2014) and assuming an average redshift $z=2.4$ for the sample here, DLAs around $[\mathrm{M} / \mathrm{H}] \approx-0.1$ are expected to have a typical stellar mass of $\sim 10^{11} M_{\odot}$, while at $[\mathrm{M} / \mathrm{H}] \approx-1.2$, this is $\sim 10^{9} M_{\odot}$. Smaller, Sculptor-like DLAs with a stellar mass of $\sim 10^{6} M_{\odot}$ should thus presumably be found at lower metallicities, $[\mathrm{S} / \mathrm{H}] \leq-2$, where data are currently lacking. A continuation of the constant $[\mathrm{S} / \mathrm{Zn}]$ trend would imply that the most metal-poor DLAs are consistent with the early stages of dwarf galaxies like Sculptor.

Comparison with DLAs is further complicated by the fact that, in general, galaxies are not detectable as DLAs through their entire evolutionary history. For example, Salvadori \& Ferrara (2012) predict that most Sculptor-like galaxies have typically already lost too much gas at $z=2.3$ (age $=10.8 \mathrm{Gyr}$ ) to be detectable as DLAs. In Fig. 6 this corresponds to $[\mathrm{S} / \mathrm{H}]>$ -1.8 , where $[\mathrm{S} / \mathrm{Zn}]$ abundance ratios are significantly higher in Sculptor than in DLAs. It is therefore plausible that some DLA galaxies may have undergone a chemical evolution similar to what we see in Sculptor, but are no longer detectable as DLAs by the time we observe them (and are therefore missing from DLA samples). How long a given galaxy retains its $\mathrm{H}$ I gas and is detectable as a DLA highly depends on its environment and star formation history.

\subsection{An alternative to $[\alpha / F e]$}

As a measurement of relative type Ia and type II SN contributions, we propose the use of the $[\mathrm{O} / \mathrm{S}]$ abundance ratio. Both these elements are volatile, and as some $S$ is produced in type Ia $\mathrm{SN}$ and $\mathrm{O}$ is essentially not, we expect the behavior of [O/S] with $[\mathrm{S} / \mathrm{H}]$ to show qualitative (but not necessarily quantitative) similarities with the more often considered trend of $[\alpha / \mathrm{Fe}]$ with $[\mathrm{Fe} / \mathrm{H}]$.

The available data for DLA systems, Sculptor, and the Milky Way disk are shown in Fig. 7. As predicted, the trend of [O/S] with $[\mathrm{S} / \mathrm{H}]$ mimics that of $[\alpha / \mathrm{Fe}]$ in Sculptor. The same is true for the Milky Way where the "turnover" to a trend of decreasing $[\mathrm{O} / \mathrm{S}]$ with increasing $[\mathrm{S} / \mathrm{H}]$ occurs at higher values than in the dwarf galaxies (as is the case for $[\alpha / \mathrm{Fe}]$ versus $[\mathrm{Fe} / \mathrm{H}]$ ). In the metal-poor halo, these values are generally supersolar 


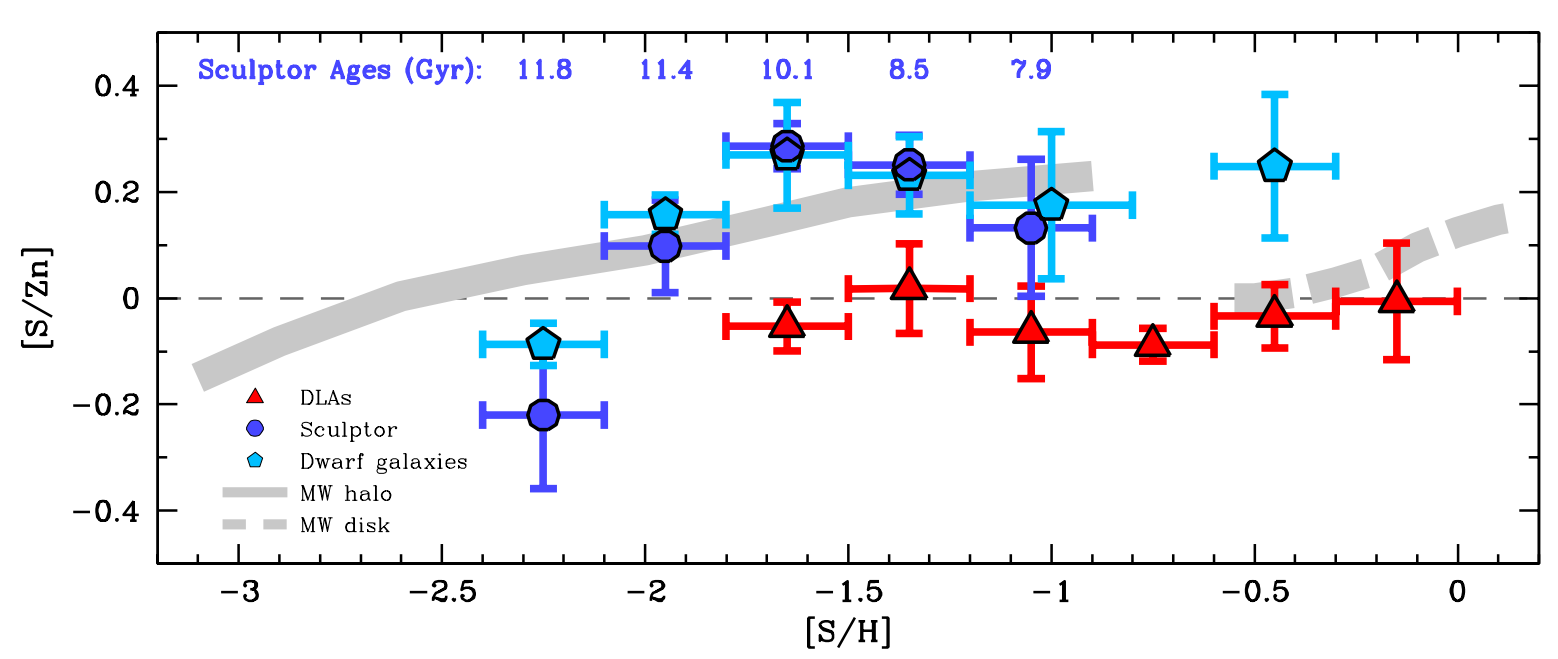

Fig. 6. Abundance ratios of S and Zn for DLAs (red triangles), Sculptor stars (blue circles) and other local dwarf galaxy stars (light blue pentagons). Average ages of Sculptor stars are listed at the top (blue) for each bin (de Boer et al. 2012). Error bars show the size of the bin (x) and the error of the mean $(y)$. Mean trends of Milky Way stars are shown with gray lines: solid for the halo (dwarf and giant stars); dashed for the disk (dwarf stars). With the exception of Sculptor, a proxy for $\mathrm{S}$ is adopted for all dwarf galaxy stars: $[\mathrm{S} / \mathrm{H}]=\frac{1}{2}([\mathrm{Ca} / \mathrm{H}]+[\mathrm{Ti} / \mathrm{H}])$. References: DLAs: Quiret et al. (2016); Dwarf galaxies: see Table 1; Milky Way halo: Cayrel et al. (2004), Nissen et al. (2004, 2007), and Spite et al. (2011); Milky Way disk: Reddy et al. (2003).

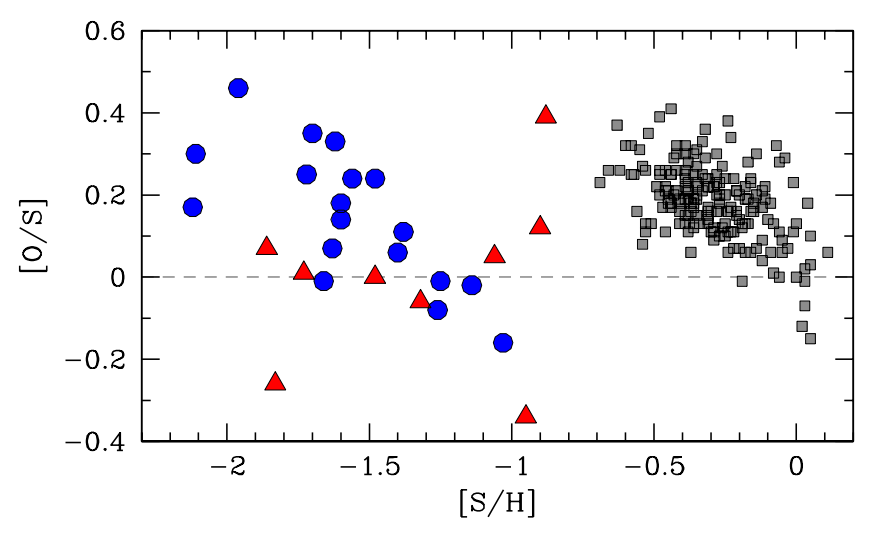

Fig. 7. The abundance ratio of $[\mathrm{O} / \mathrm{S}]$ as a function of $[\mathrm{S} / \mathrm{H}]$ for DLAs (red triangles), Sculptor stars (blue circles) and Milky Way stars (gray squares). References: DLAs: Quiret et al. (2016). Sculptor: Skúladóttir et al. (2015a) and Hill et al. (in prep.) Milky Way: Reddy et al. (2003).

with $\langle[\mathrm{O} / \mathrm{S}]\rangle=0.46 \pm 0.14$ (Cayrel et al. 2004; Spite et al. 2011).

High $[\mathrm{O} / \mathrm{S}]$ abundance ratios in DLAs should therefore correspond to systems that have not experienced a significant contribution by type Ia SN, while those with low $[\mathrm{O} / \mathrm{S}]$ have. Since the timescales of type Ia SN are $\approx 1-2 \mathrm{Gyr}$, DLAs observed at redshifts $z>3$ should have supersolar $[\mathrm{O} / \mathrm{S}]$ ratios, and the same is expected at $[\mathrm{S} / \mathrm{H}]<-2$, from what is observed in the Milky Way halo and its satellites. Unfortunately the available measurements of $[\mathrm{O} / \mathrm{S}]$ in DLA systems are very limited, mainly since $\mathrm{O}$ is challenging to measure at high metallicities, and $\mathrm{S}$ at low. Furthermore these elements are typically measured from different ionization states, O I and S II lines, possibly causing further complications.

Theoretical yields (e.g., Tsujimoto et al. 1995) predict a similar behaviour of $[\mathrm{O} / \mathrm{Si}]$ versus $[\mathrm{Si} / \mathrm{H}]$, and this is supported with Milky Way data (see Appendix A). However, $[\mathrm{O} / \mathrm{Si}]$ is expected to be slightly less sensitive to type Ia SN contributions compared to $[\mathrm{O} / \mathrm{S}]$. With the use of $\mathrm{Si}$ also comes the added complication of dust depletion in DLAs. However, in the sample of metal-poor
DLAs (where dust depletion is less effective) from Cooke et al. (2011), supersolar values are observed: $\langle[\mathrm{O} / \mathrm{Si}]\rangle=0.11 \pm 0.06$ at $-3<[\mathrm{Si} / \mathrm{H}]<-2$. This is consistent with only moderate type Ia SN contribution. In comparison, the metal-poor Milky Way halo has $\langle[\mathrm{O} / \mathrm{Si}]\rangle=0.30 \pm 0.11$ at similar metallicities (Cayrel et al. 2004).

\section{Consequences for previous studies}

Other studies have previously tried to use the volatile elements $\mathrm{S}$ and $\mathrm{Zn}$ to make comparisons between the chemical properties of DLAs and stars in the Local Group (e.g., Nissen et al. 2007; Spite et al. 2011). In particular, the link between DLAs and local dwarf galaxies was explored in detail in recent work by Cooke et al. (2015) and Berg et al. (2015). However, the chemical abundance comparison in these studies was somewhat limited by lack of data in Local Group dwarf galaxies, as very few $[\mathrm{Zn} / \mathrm{H}]$ and no $[\mathrm{S} / \mathrm{Zn}]$ measurements were available. Both Berg et al. (2015) and Cooke et al. (2015) therefore used $[\mathrm{Zn} / \mathrm{H}]$ and $[\mathrm{Fe} / \mathrm{H}]$ interchangeably.

The main new insight provided by the [S/Zn] data in Sculptor is the complicated nature of $\mathrm{Zn}$ abundances in dwarf galaxies, as captured in Fig. 4. Using $[\mathrm{Zn} / \mathrm{H}]$ as a proxy for $[\mathrm{Fe} / \mathrm{H}]$ therefore leads to significant differences in chemical patterns. To illustrate this effect, schematic views of the mean trends of [O/Fe], a typical $[\alpha / \mathrm{Fe}]$ tracer, and $[\mathrm{S} / \mathrm{Zn}]$ abundance ratios in Sculptor are shown both as a function of $[\mathrm{Fe} / \mathrm{H}]$ and $[\mathrm{Zn} / \mathrm{H}]$ in Fig. 8. These abundance ratios show significantly different trends, both from each other, and whether $[\mathrm{Fe} / \mathrm{H}]$ or $[\mathrm{Zn} / \mathrm{H}]$ is used as a reference element. In conclusion: [S/Zn] does not show a similar relation with metallicity as $[\mathrm{O} / \mathrm{Fe}]$, or other $[\alpha / \mathrm{Fe}]$ ratios do. Relevant discussion in previous comparison studies should therefore be revisited.

In particular, Cooke et al. (2015) showed [O/Fe] as a function of $[\mathrm{Fe} / \mathrm{H}]$ for $[\mathrm{Fe} / \mathrm{H}] \lesssim-2$, and $[\mathrm{S} / \mathrm{Zn}]$ as a function of $[\mathrm{Zn} / \mathrm{H}]$ for $[\mathrm{Fe} / \mathrm{H}] \gtrsim-2$, assuming that both abundance ratios were tracing $[\alpha / \mathrm{Fe}]$ versus $[\mathrm{Fe} / \mathrm{H}]$ in DLAs. By doing this, they observed a 'knee' in these abundance ratios where they started to decrease at $[\mathrm{Fe} / \mathrm{H}] \approx-2$, analogous to that observed in $[\alpha / \mathrm{Fe}]$ as a 

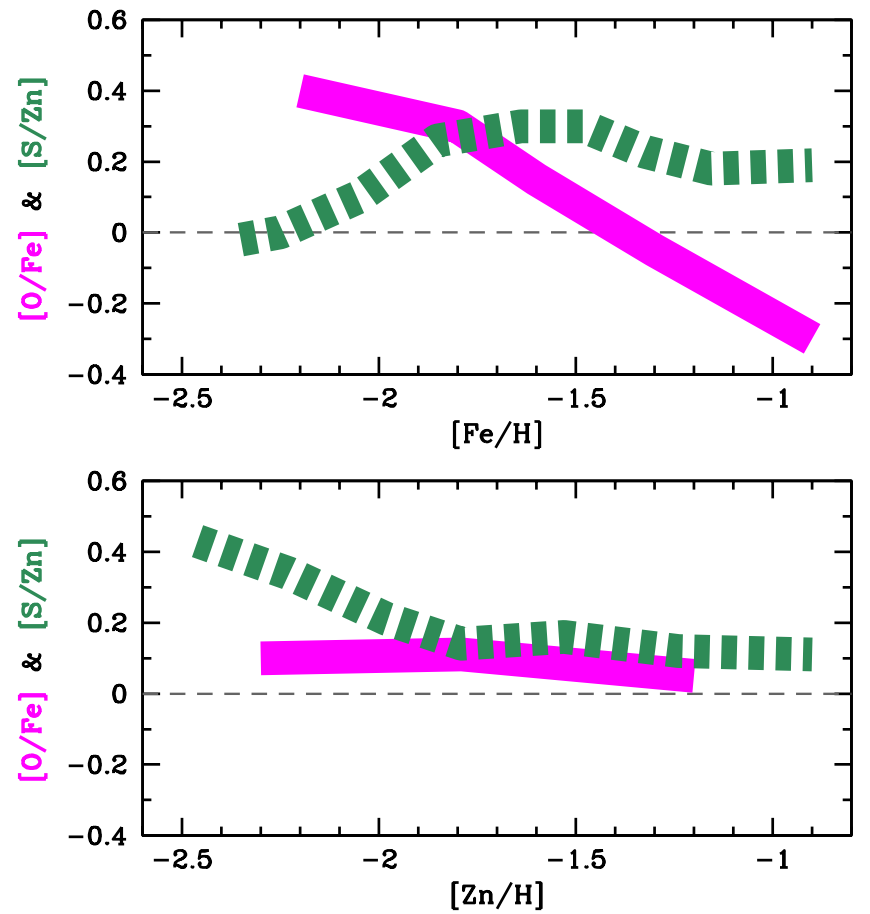

Fig. 8. Schematic view of the mean trends of $[\mathrm{O} / \mathrm{Fe}]$ (magenta solid lines) and $[\mathrm{S} / \mathrm{Zn}]$ (green dashed lines) in the Sculptor $\mathrm{dSph}$ as a function of $[\mathrm{Fe} / \mathrm{H}]($ top $)$ and $[\mathrm{Zn} / \mathrm{H}]($ bottom). References: $[\mathrm{O} / \mathrm{Fe}]$ : Hill et al. in prep. [S/Zn]: Skúladóttir et al. (2015a, 2017).

function of $[\mathrm{Fe} / \mathrm{H}]$ in dwarf galaxies. However, it is clear from Fig. 8 that this assumption does not hold (see also Sect. 4). Therefore, although a 'knee' in $[\alpha / \mathrm{Fe}]$ abundance ratios in DLAs cannot be excluded at this point, further confirmation is needed, preferably using the same probe throughout the entire metallicity range.

\section{Conclusions}

For the first time we have made a detailed chemical comparison between DLAs and stars in dwarf galaxies by using the volatile elements $\mathrm{S}$ and $\mathrm{Zn}$ without the help of any other element as proxy. This was made possible by recent measurements of $\mathrm{S}$ and $\mathrm{Zn}$ abundances in around 80 RGB stars in the Sculptor dwarf spheroidal galaxy (Skúladóttir et al. 2015a, 2017). Most of the stars in this galaxy formed $>10 \mathrm{Gyr}$ ago, which correspond to the redshifts at which the majority of DLAs are observed (i.e., $z \gtrsim 1.8$ ).

Our analysis of the Sculptor data shows that $\mathrm{S}$ abundances behave as expected of an $\alpha$-element, primarily created by corecollapse SN. However, there is also a non-negligible contribution to $\mathrm{S}$ from the yields of type Ia $\mathrm{SN}$. This makes $[\mathrm{S} / \mathrm{H}]$ a reasonable tracer of the metallicity evolution in galaxies. In low-metallicity stars, S measurements are challenging, so when not available, other $\alpha$-elements can be used as proxy, preferably: $\mathrm{Ca}, \mathrm{Ti}$ or the average of the two (see Appendix A). The lighter $\alpha$-elements, $\mathrm{O}$ and $\mathrm{Mg}$, should not be used as proxies for $\mathrm{S}$ since, unlike $\mathrm{S}$, they have negligible contribution from type Ia SN.

In light of the available measurements in the Milky Way and its satellite dwarf galaxies, it is clear that $[\mathrm{Zn} / \mathrm{Fe}]$ is neither the same at a given metallicity in all galaxies probed here, nor constant in a given galaxy throughout its entire evolutionary history. The nucleosynthetic channels that lead to the formation of $\mathrm{Zn}$ remain unclear, but the following statement is in agreement with both theory and observations: Type Ia SN are not significant producers of $\mathrm{Zn}$. The formation channels of $\mathrm{Zn}$ are therefore not the same as $\mathrm{Fe}$, and the $[\mathrm{Zn} / \mathrm{Fe}]$ ratio is highly dependent on the star formation history of each system. Thus, using $[\mathrm{Zn} / \mathrm{H}]$ as a proxy for $[\mathrm{Fe} / \mathrm{H}]$ is fraught with potential pitfalls.

Furthermore, $[\mathrm{S} / \mathrm{Zn}]$ should not be used as a proxy for $[\alpha / \mathrm{Fe}]$, which is commonly interpreted as indicative of the relative contributions from core-collapse (such as type II SN) and type Ia $\mathrm{SN}$. Instead we suggest $[\mathrm{O} / \mathrm{S}]$ with $[\mathrm{S} / \mathrm{H}]$, which shows qualitatively the same trend as $[\alpha / \mathrm{Fe}]$ with $[\mathrm{Fe} / \mathrm{H}]$ both in the Sculptor dwarf spheroidal galaxy and in the Milky Way. Both $\mathrm{O}$ and $\mathrm{S}$ are volatile elements that are not expected to be significantly depleted onto dust, and $\mathrm{O}$ traces mainly massive stars exploding as type II SN, while $\mathrm{S}$ is formed both in type Ia and II SN. We therefore predict supersolar [O/S] in DLAs at low metallicities, $[\mathrm{S} / \mathrm{H}]<-2$, and in particular at $z>3$, as type Ia SN have not yet had time to significantly enrich the environment.

Assuming that dust depletion of $\mathrm{S}$ and $\mathrm{Zn}$ in DLAs is negligible, we conclude that DLAs on average do not have both the same $[\mathrm{S} / \mathrm{Fe}]$ and $[\mathrm{Zn} / \mathrm{Fe}]$ as a function of $[\mathrm{Zn} / \mathrm{H}]$ as either the Milky Way or the Sculptor dwarf spheroidal galaxy. Furthermore, over the full range of $[\mathrm{S} / \mathrm{H}]$ metallicities considered here, $-2 \lesssim[\mathrm{S} / \mathrm{H}] \lesssim 0$, the $[\mathrm{S} / \mathrm{Zn}]$ relative abundances of DLAs do not resemble the patterns found in either the Milky Way nor its dwarf galaxy satellites. Thus, DLA systems on average do not have the same star formation history at all metallicities, as the Milky Way nor Sculptor, nor any other dwarf galaxy where data are sufficient. This should not be surprising, as DLA systems are likely a mixed bag of objects, possibly with different properties at different metallicities. Therefore there is no reason to expect their star formation history to always follow that of the Milky Way or any other galaxy.

Considering the different formation scenarios of $\mathrm{S}$ and $\mathrm{Zn}$, and the complexity observed in stars in the Local Group galaxies, it is remarkable that the $[\mathrm{S} / \mathrm{Zn}]$ ratio is on average constant at approximately the solar value over the full metallicity range probed by DLAs, $-1.8 \lesssim[\mathrm{S} / \mathrm{H}] \lesssim 0$ (see Fig. 6). It is possible that this is simply the result of averaging over galaxies with different star formation histories in each metallicity bin, or that we are looking at different galaxies at a similar stage in their evolution. Until we gather more information about the host galaxies of DLAs and the nucleosynthesis of $\mathrm{Zn}$ in particular, the lack of any trend in $[\mathrm{S} / \mathrm{Zn}]$ versus $[\mathrm{S} / \mathrm{H}]$ in DLAs remains a puzzle.

Finally, we note that although $\mathrm{Zn}$ is not interchangeable with $\mathrm{Fe}$, it can still be reliably used as a metallicity indicator of DLA systems. The lowest relative $[\mathrm{Zn} / \mathrm{Fe}]$ abundances we have measured in the Local Group are in galaxies that have lost their gas, and the highest $[\mathrm{Zn} / \mathrm{Fe}]$ are measured at very low $[\mathrm{Fe} / \mathrm{H}]$, where $\mathrm{Zn}$ is very challenging to measure in gas (see Fig. 4). It is therefore unlikely that these extremes are very common in DLAs. Away from such extremes, Zn should be a reasonably good metallicity indicator.

Acknowledgements. The authors are indebted to the International Space Science Institute (ISSI), Bern, Switzerland, for supporting and funding the international team "First stars in dwarf galaxies". Á.S. thanks S. Quiret for providing his DLA literature compilation, R. Cooke for insightful suggestions, and P. Noterdaeme for useful help. Á.S. acknowledges funds from the Alexander von Humboldt Foundation in the framework of the Sofja Kovalevskaja Award endowed by the Federal Ministry of Education and Research. E.T. gratefully acknowledges support to visit to the IoA from the Sackler Fund for Astronomy. S.S. was supported by the European Commission through a Marie-Curie Fellowship, project PRIMORDIAL, grant nr. 700907, and by the Italian Ministry of Education, University, and Research (MIUR) through a Rita Levi Montalcini Fellowship. M.P. is grateful to the Kapteyn Astronomical Institute for their generous hospitality during multiple visits while this work was in progress. 


\section{References}

Akerman, C. J., Ellison, S. L., Pettini, M., \& Steidel, C. C. 2005, A\&A, 440, 499 Aparicio, A., Carrera, R., \& Martínez-Delgado, D. 2001, AJ, 122, 2524

Barbuy, B., Friaça, A. C. S., da Silveira, C. R., et al. 2015, A\&A, 580, A40

Bechtold, J. 2003, in Galaxies at High Redshift, eds. I. Pérez-Fournon, M. Balcells, F. Moreno-Insertis, \& F. Sánchez (Cambridge, UK: Cambridge University Press), 131

Becker, G. D., Bolton, J. S., \& Lidz, A. 2015, PASA, 32, 45

Bellazzini, M., Correnti, M., Ferraro, F. R., Monaco, L., \& Montegriffo, P. 2006, A\&A, 446, L1

Bensby, T., Yee, J. C., Feltzing, S., et al. 2013, A\&A, 549, A147

Bensby, T., Feltzing, S., \& Oey, M. S. 2014, A\&A, 562, A71

Berg, T. A. M., Ellison, S. L., Prochaska, J. X., Venn, K. A., \& DessaugesZavadsky, M. 2015, MNRAS, 452, 4326

Bird, S., Haehnelt, M., Neeleman, M., et al. 2015, MNRAS, 447, 1834

Boylan-Kolchin, M., Weisz, D. R., Johnson, B. D., et al. 2015, MNRAS, 453, 1503

Caffau, E., Ludwig, H.-G., Steffen, M., Freytag, B., \& Bonifacio, P. 2011 Sol. Phys., 268, 255

Carollo, D., Beers, T. C., Placco, V. M., et al. 2016, Nat. Phys., 12, 1170

Cayrel, R., Depagne, E., Spite, M., et al. 2004, A\&A, 416, 1117

Chen, Y. Q., Nissen, P. E., Zhao, G., \& Asplund, M. 2002, A\&A, 390, 225

Chen, Y. Q., Zhao, G., Nissen, P. E., Bai, G. S., \& Qiu, H. M. 2003, ApJ, 591, 925

Christensen, L., Møller, P., Fynbo, J. P. U., \& Zafar, T. 2014, MNRAS, 445, 225

Cohen, J. G., \& Huang, W. 2009, ApJ, 701, 1053

Cohen, J. G., \& Huang, W. 2010, ApJ, 719, 931

Cooke, R., Pettini, M., Steidel, C. C., Rudie, G. C., \& Nissen, P. E. 2011 MNRAS, 417, 1534

Cooke, R. J., Pettini, M., \& Jorgenson, R. A. 2015, ApJ, 800, 12

de Boer, T. J. L., Tolstoy, E., Hill, V., et al. 2012, A\&A, 539, A103

de Boer, T. J. L., Belokurov, V., \& Koposov, S. 2015, MNRAS, 451, 3489

De Cia, A., Ledoux, C., Mattsson, L., et al. 2016, A\&A, 596, A97

Duffau, S., Caffau, E., Sbordone, L., et al. 2017, A\&A, 604, A128

Fumagalli, M., O’Meara, J. M., Prochaska, J. X., Rafelski, M., \& Kanekar, N. 2015, MNRAS, 446, 3178

Geisler, D., Smith, V. V., Wallerstein, G., Gonzalez, G., \& Charbonnel, C. 2005, AJ, 129, 1428

Gilmore, G., Norris, J. E., Monaco, L., et al. 2013, ApJ, 763, 61

Grevesse, N., \& Sauval, A. J. 1998, Space Sci. Rev., 85, 161

Heger, A., \& Woosley, S. E. 2002, ApJ, 567, 532

Hirai, Y., Saitoh, T. R., Ishimaru, Y., \& Wanajo, S. 2018, ApJ, 855, 63

Hoffman, R. D., Woosley, S. E., Fuller, G. M., \& Meyer, B. S. 1996, ApJ, 460, 478

Honda, S., Aoki, W., Arimoto, N., \& Sadakane, K. 2011, PASJ, 63, 523

Ishigaki, M. N., Aoki, W., \& Chiba, M. 2013, ApJ, 771, 67

Iwamoto, K., Brachwitz, F., Nomoto, K., et al. 1999, ApJS, 125, 439

Jenkins, E. B. 2009, ApJ, 700, 1299

Jenkins, E. B., \& Wallerstein, G. 2017, ApJ, 838, 85

Jorgenson, R. A., Murphy, M. T., \& Thompson, R. 2013, MNRAS, 435, 482

Kirby, E. N., \& Cohen, J. G. 2012, AJ, 144, 168

Kirby, E. N., Cohen, J. G., Guhathakurta, P., et al. 2013, ApJ, 779, 102

Kobayashi, C., Umeda, H., Nomoto, K., Tominaga, N., \& Ohkubo, T. 2006, ApJ, 653,1145

Korotin, S. A. 2008, Odessa Astron. Publ., 21, 42

Krogager, J.-K., Møller, P., Fynbo, J. P. U., \& Noterdaeme, P. 2017, MNRAS 469, 2959

Lee, H., Skillman, E. D., Cannon, J. M., et al. 2006, ApJ, 647, 970

Lemasle, B., Hill, V., Tolstoy, E., et al. 2012, A\&A, 538, A100

Madau, P., \& Dickinson, M. 2014, ARA\&A, 52, 415

Mas-Ribas, L., Miralda-Escudé, J., Pérez-Ràfols, I., et al. 2017, ApJ, 846, 4

Matteucci, F., \& Greggio, L. 1986, A\&A, 154, 279

McConnachie, A. W. 2012, AJ, 144, 4
Mikolaitis, $\breve{S}$., de Laverny, P., Recio-Blanco, A., et al. 2017, A\&A, 600, A22 Mishenina, T. V., Kovtyukh, V. V., Soubiran, C., Travaglio, C., \& Busso, M. 2002, A\&A, 396, 189

Neeleman, M., Prochaska, J. X., Ribaudo, J., et al. 2016, ApJ, 818, 113

Nissen, P. E., \& Schuster, W. J. 2010, A\&A, 511, L10

Nissen, P. E., \& Schuster, W. J. 2011, A\&A, 530, A15

Nissen, P. E., Chen, Y. Q., Asplund, M., \& Pettini, M. 2004, A\&A, 415, 993

Nissen, P. E., Akerman, C., Asplund, M., et al. 2007, A\&A, 469, 319

Nomoto, K., Kobayashi, C., \& Tominaga, N. 2013, ARA\&A, 51, 457

Péroux, C., Bouché, N., Kulkarni, V. P., York, D. G., \& Vladilo, G. 2012, MNRAS, 419, 3060

Pettini, M. 2004, in Cosmochemistry. The Melting Pot of the Elements, eds. C. Esteban, R. García López, A. Herrero, \& F. Sánchez (Cambridge, UK: Cambridge University Press), 257

Pettini, M., \& Cooke, R. J. 2014, Mem. Soc. Astron. It., 85, 542

Pettini, M., Boksenberg, A., \& Hunstead, R. W. 1990, ApJ, 348, 48

Planck Collaboration XIII. 2016, A\&A, 594, A13

Pontzen, A., Governato, F., Pettini, M., et al. 2008, MNRAS, 390, 1349

Pruet, J., Woosley, S. E., Buras, R., Janka, H.-T., \& Hoffman, R. D. 2005, ApJ, 623,325

Quiret, S., Péroux, C., Zafar, T., et al. 2016, MNRAS, 458, 4074

Rafelski, M., Wolfe, A. M., Prochaska, J. X., Neeleman, M., \& Mendez, A. J. 2012, ApJ, 755, 89

Rafelski, M., Neeleman, M., Fumagalli, M., Wolfe, A. M., \& Prochaska, J. X. 2014, ApJ, 782, L29

Reddy, B. E., Tomkin, J., Lambert, D. L., \& Allende Prieto C. 2003, MNRAS, 340,304

Reddy, B. E., Lambert, D. L., \& Allende Prieto C. 2006, MNRAS, 367, 1329

Sadakane, K., Arimoto, N., Ikuta, C., et al. 2004, PASJ, 56, 1041

Salvadori, S., \& Ferrara, A. 2012, MNRAS, 421, L29

Salvadori, S., Ferrara, A., Schneider, R., Scannapieco, E., \& Kawata, D. 2010, MNRAS, 401, L5

Sánchez-Ramírez, R., Ellison, S. L., Prochaska, J. X., et al. 2016, MNRAS, 456, 4488

Savage, B. D., \& Sembach, K. R. 1996, ARA\&A, 34, 279

Sbordone, L., Bonifacio, P., Buonanno, R., et al. 2007, A\&A, 465, 815

Shetrone, M., Venn, K. A., Tolstoy, E., et al. 2003, AJ, 125, 684

Shetrone, M. D., Côté, P., \& Sargent, W. L. W. 2001, ApJ, 548, 592

Siegel, M. H., Dotter, A., Majewski, S. R., et al. 2007, ApJ, 667, L57

Simon, J. D., Jacobson, H. R., Frebel, A., et al. 2015, ApJ, 802, 93

Skillman, E. D., Kennicutt, R. C., \& Hodge, P. W. 1989, ApJ, 347, 875

Skúladóttir, Á., Andrievsky, S. M., Tolstoy, E., et al. 2015a, A\&A, 580, A129

Skúladóttir, Á., Tolstoy, E., Salvadori, S., et al. 2015b, A\&A, 574, A129

Skúladóttir, Á., Tolstoy, E., Salvadori, S., Hill, V., \& Pettini, M. 2017, A\&A, 606, A71

Spite, M., Caffau, E., Andrievsky, S. M., et al. 2011, A\&A, 528, A9

Takeda, Y., Hashimoto, O., Taguchi, H., et al. 2005, PASJ, 57, 751

Tchernyshyov, K., Meixner, M., Seale, J., et al. 2015, ApJ, 811, 78

Tolstoy, E., Hill, V., \& Tosi, M. 2009, ARA\&A, 47, 371

Travaglio, C., Gallino, R., Arnone, E., et al. 2004, ApJ, 601, 864

Tsujimoto, T., Nomoto, K., Yoshii, Y., et al. 1995, MNRAS, 277, 945

Umeda, H., \& Nomoto, K. 2002, ApJ, 565, 385

Ural, U., Cescutti, G., Koch, A., et al. 2015, MNRAS, 449, 761

Venn, K. A., Shetrone, M. D., Irwin, M. J., et al. 2012, ApJ, 751, 102

Vladilo, G. 2002, ApJ, 569, 295

Wanajo, S., Müller, B., Janka, H.-T., \& Heger, A. 2018, ApJ, 852, 40

Wheeler, J. C., Sneden, C., \& Truran, Jr., J. W. 1989, ARA\&A, 27, 279

Wise, J. H., Demchenko, V. G., Halicek, M. T., et al. 2014, MNRAS, 442, 2560

Wolfe, A. M., Gawiser, E., \& Prochaska, J. X. 2005, ARA\&A, 43, 861

Wolfire, M. G., McKee, C. F., Hollenbach, D., \& Tielens, A. G. G. M. 2003, ApJ, 587,278

Wright, E. L. 2006, PASP, 118, 1711

Wyse, R. F. G., \& Gilmore, G. 1988, AJ, 95, 1404 


\section{Appendix A: Proxy for sulphur in stars}

The available $\mathrm{S}$ measurements in stars in dwarf galaxies are relatively scarce (see Table 1). Therefore, it is tempting to try to find another $\alpha$-element in stars that is suited for comparison with $\mathrm{S}$ in DLA systems. Comparisons of sulphur with other $\alpha$ element measurements in Sculptor and the Milky Way are shown in Fig. A.1. Notably, not all $[\mathrm{S} / \alpha]$ abundance ratios show the same trends with $[\mathrm{Fe} / \mathrm{H}]$, in either system. Larger deviations from a flat $[\mathrm{S} / \alpha]$ abundance ratio make the $\alpha$-element in question less suited to be used as a proxy for sulphur.

As already reported in Skúladóttir et al. (2015a), [S/Mg] in Sculptor shows an increasing trend with $[\mathrm{Fe} / \mathrm{H}]$, and the same is true for $[\mathrm{S} / \mathrm{O}]$. This is consistent with theoretical yields which predict some production of $\mathrm{S}$ by type Ia $\mathrm{SN}$, but negligible contribution to both $\mathrm{O}$ and $\mathrm{Mg}$ (e.g., Tsujimoto et al. 1995; Iwamoto et al. 1999). Thus the ratios of $[\mathrm{S} / \mathrm{Mg}]$ and $[\mathrm{S} / \mathrm{O}]$ are higher in the yields of type Ia SN compared to type II SN, so as the pollution by the former becomes more significant, the values of these abundance ratios increase. The trend in Sculptor of these ratios is therefore opposite to that of $[\alpha / \mathrm{Fe}]$ ratios observed at the same metallicity in this galaxy. Also in agreement with this explanation, $[\mathrm{S} / \mathrm{O}]$ and $[\mathrm{S} / \mathrm{Mg}]$ are not the same in the metal-poor halo and in Milky Way disk stars, confirming observationally that $\mathrm{O}$ and $\mathrm{Mg}$ are not ideal proxies for $\mathrm{S}$.

There are too few measurements of Si in Sculptor to draw any strong conclusions of how well it traces S. However, although there is only one data point at $[\mathrm{Fe} / \mathrm{H}]=-2$ this measurement is from higher-quality data (higher resolution and longer wavelength range) compared to the other Si measurement in Sculptor (Skúladóttir et al. 2015b, Hill et al. in prep). By trusting this one data point, the trend with $[\mathrm{Fe} / \mathrm{H}]$ seems weak, suggesting that $\mathrm{Si}$ is at least a better option than both $\mathrm{O}$ and $\mathrm{Mg}$. The differences of [S/Si] at the metal-rich and metal-poor ends in the Milky Way are small, and seem to indicate that $\mathrm{Si}$ traces $\mathrm{S}$ reasonably well. This is consistent with theory. Tsujimoto et al. (1995) computed the relative contributions by type Ia and II SN for various elements and found that $25 \%$ of S and $17 \%$ of $\mathrm{Si}$ in the solar neighborhood came from type Ia SN (while it was $1 \%$ for both $\mathrm{O}$ and $\mathrm{Mg}$ ).

The elements $\mathrm{Ca}$ and $\mathrm{Ti}$ seem to trace $\mathrm{S}$ reasonably, both in Sculptor and the Milky Way, with $-0.2<[\mathrm{S} / \mathrm{Ca}]<0.1$ and $-0.1<[\mathrm{S} / \mathrm{Ti}]<0.2$. Both these elements have the advantage that they are relatively easy to measure in stars as there are many available lines in the optical part of the spectrum. There is a minor increasing trend in Sculptor of [S/Ti] with metallicity, while $[\mathrm{S} / \mathrm{Ca}]$ is consistent with a flat, or a slightly decreasing trend. Tsujimoto et al. (1995) predicted that $25 \%$ of $\mathrm{Ca}$ in the solar neighborhood originates in type Ia SN, which is exactly the same value as for $\mathrm{S}$. Their study did not include $\mathrm{Ti}$, but it has proven difficult to calculate theoretical Ti yields that fit observational data (e.g., Kobayashi et al. 2006). However, observationally, both these elements seem to trace $S$ reasonably well. Another possibility is to use the average of the two elements, and in all cases $-0.1<[\mathrm{S} /(\mathrm{Ca}+\mathrm{Ti})]<0.1$, where the ratio is defined as $\frac{1}{2}([\mathrm{~S} / \mathrm{Ca}]+[\mathrm{S} / \mathrm{Ti}])$; see bottom panels of Fig. A.1. Using two elements also has the advantage of reducing measurement errors.
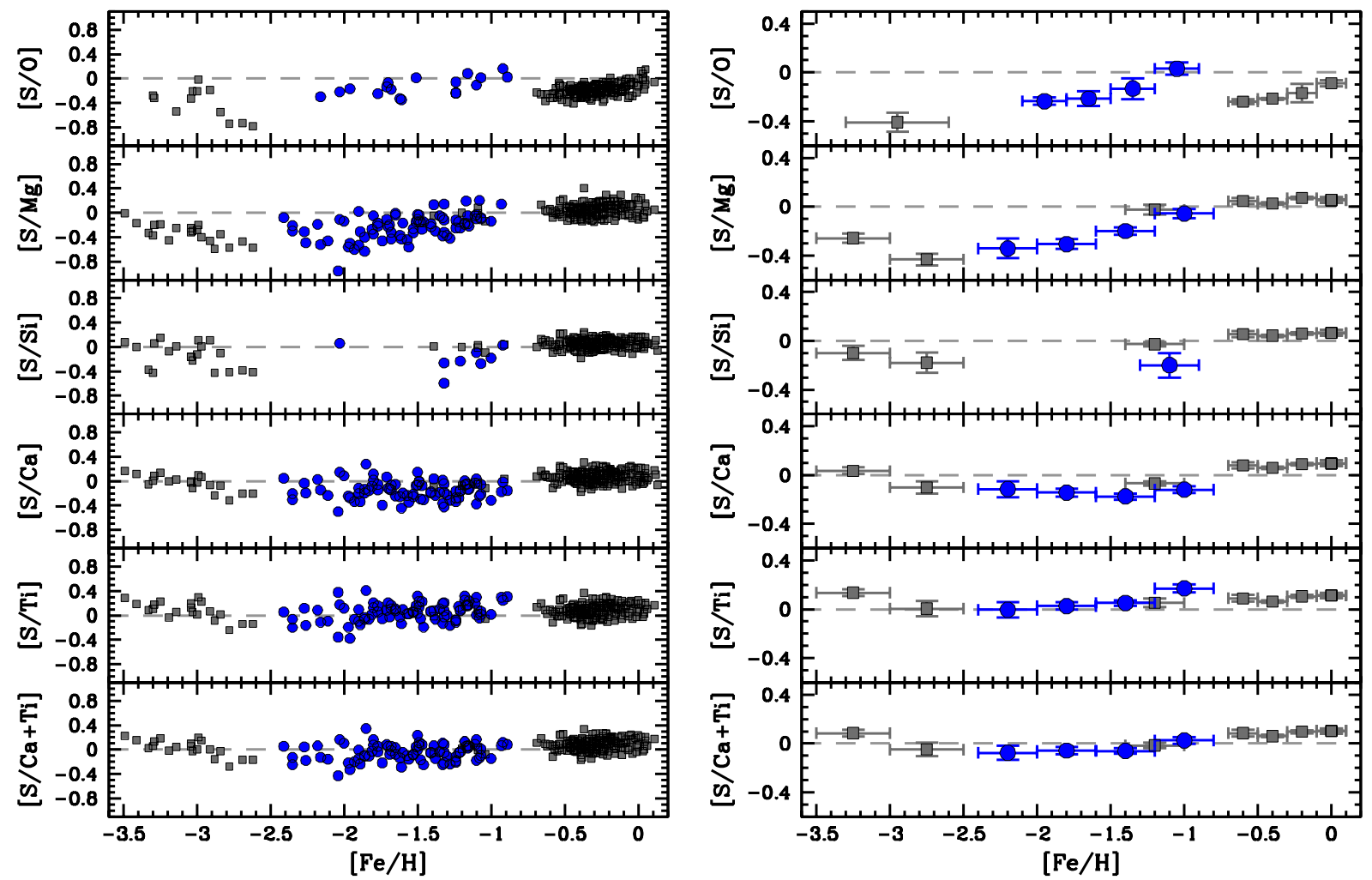

Fig. A.1. Stellar measurements in Sculptor (blue circles) and the Milky Way (gray squares) of abundance ratios of sulphur to other $\alpha$-elements: O, $\mathrm{Mg}, \mathrm{Si}, \mathrm{Ca}, \mathrm{Ti}$, and $[\mathrm{S} /(\mathrm{Ca}+\mathrm{Ti})]=\frac{1}{2}([\mathrm{~S} / \mathrm{Ca}]+[\mathrm{S} / \mathrm{Ti}])$. Left panels show individual measurements while right panels show binned data, where the $x$-axis error bar shows the size of each bin and y-axis error bar is the error of mean. We note that the range on the $y$-axis is not the same in left and right panels. References: Sculptor: Skúladóttir et al. (2015b) (1 star), Skúladóttir et al. (2015a) (S), Skúladóttir et al. (2017) (Ti), Hill et al. in prep (Mg, Si, Ca). Milky Way: Chen et al. (2002), Reddy et al. (2003), Chen et al. (2003), Cayrel et al. (2004), Nissen et al. (2007), Nissen \& Schuster (2010), and Spite et al. (2011). 
It is important to keep in mind that each tracer has its own limitations and that $\mathrm{Ca}$ and $\mathrm{Ti}$ are not completely equivalent. In Sculptor, $[\mathrm{Ca} / \mathrm{Ti}]$ shows a weak increasing trend with $[\mathrm{Fe} / \mathrm{H}]$, indicating that the ratio $[\mathrm{Ca} / \mathrm{Ti}]$ is not the same in type Ia $\mathrm{SN}$ as in type II SN, and/or that the metallicity dependence of the yields of these two elements is different. A similar result is seen in the Milky Way halo, where Nissen \& Schuster (2010) reported two distinct halo populations in the solar neighborhood, with different values of $[\alpha / \mathrm{Fe}]$. Similar to Sculptor, the halo stars with low- $\alpha$ have a high value of $\langle[\mathrm{Ca} / \mathrm{Ti}]\rangle_{\text {low- } \alpha}$
$=0.098 \pm 0.006$, compared to the high- $\alpha$ population, $\langle[\mathrm{Ca} / \mathrm{Ti}]\rangle_{\text {high }-\alpha}=0.042 \pm 0.005$. When comparing stellar and DLA abundances it is thus advised to consistently use the same proxy for $\mathrm{S}$ in all the stars involved, to decrease differences in systematic errors.

In conclusion, $S$ in DLA systems should ideally be compared to $\mathrm{S}$ in stars. When this is not an option, using $\mathrm{Ca}$, $\mathrm{Ti}$, or the average of the two compared to the solar value should be the best alternative, judging from the available data in Sculptor and the Milky Way. 